\title{
The Weighted Mean Standard Deviation Distribution: A Geometrical Framework
}

\author{
R. Caimmi \\ Physics and Astronomy Department, Padua University, Padova, Italy \\ Email: roberto.caimmi@unipd.it
}

Received 23 February 2015; accepted 11 March 2015; published 12 March 2015

Copyright (C) 2015 by author and Scientific Research Publishing Inc.

This work is licensed under the Creative Commons Attribution International License (CC BY). http://creativecommons.org/licenses/by/4.0/

(c) (†) Open Access

\section{Abstract}

The current attempt is aimed to extend previous results, concerning the explicit expression of the arithmetic mean standard deviation distribution, to the general case of the weighted mean standard deviation distribution. To this respect, the integration domain is expressed in canonical form after a change of reference frame in the $n$-space, which is recognized as an infinitely thin $n$-cylindrical corona where the axis coincides with a coordinate axis and the orthogonal section is an infinitely thin, homotetic $(n-1)$-elliptical corona. The semiaxes are formulated in two different ways, namely in terms of (1) eigenvalues, via the eigenvalue equation, and (2) leading principal minors of the matrix of a quadratic form, via the Jacobi formulae. The distribution and related parameters have the same formal expression with respect to their counterparts in the special case where the weighted mean coincides with the arithmetic mean. The reduction of some results to ordinary geometry is also considered.

\section{Keywords}

Standard Deviation, $n$-Spaces, Direction Cosines, Quadratic Forms, Matrix Theory

\section{Introduction}

Hyperspace geometry is useful, if not essential, for the interpretation of theories involving many branches of knowledge and, in particular, general relativity (e.g., [1]) and superstring theory (e.g., [2]). Though further insight could be gained exploiting a geometrical framework, still pure mathematical analysis is preferred owing to a much greater difficulty in handling with hyperspace geometry.

The geometrical framework of a well known statistical problem, concerning the explicit expression of the arithmetic mean standard deviation distribution, has been considered in an earlier investigation [3].

The current attempt is aimed at extenting the above mentioned results to the general case of the weighted 
mean, or in other words searching the explicit expression of the weighted mean standard deviation distribution, under the safely motivated restriction of independent measures obeying Gaussian distributions. Accordingly, the weighted mean standard deviation depends only on the uncertainties on the input data, which is not in contradiction with its counterpart inferred from a different approach based on the least-squares method [4].

The extension of the procedure followed in the parent paper [3] yields results which reduce to their counterparts therein, in the special case where the weighted mean reduces to the arithmetic mean.

The paper is organized as follows. The problem is outlined in Section 2 together with three steps towards the solution. The first, second, third step are exploited in Sections 3, 4, 5, respectively. In addition, three different attempts are exploited in Section 4. The solution of the problem is shown in Section 6, where a number of (well known) related parameters are also inferred for sake of completeness. The conclusion is drawn in Section 7. Further details are shown in the Appendix, including extension of analytic geometry formulation to $(n+1)$ spaces, Jacobi formulae, reduction of the results to ordinary geometry, and a corrigendum to the parent paper [3].

\section{The Problem}

Let $f_{j}\left(m_{j k}\right) \mathrm{d} m_{j k}$ be distributions related to assigned measure methods, $\alpha_{j}, 1 \leq j \leq n^{\prime}$, and a specified statistical system, where the occurrence of the events, $E_{j k}$, has been designed by the value of the random variables, $m_{j k}, 1 \leq k \leq n_{j}$. The special case of Gaussian distributions, which well holds for independent measures, reads:

$$
f_{j}\left(m_{j k}\right) \mathrm{d} m_{j k}=\frac{1}{\sqrt{2 \pi} \sigma_{m_{j}}} \exp \left[-\frac{\left[m_{j k}-\left(m_{j}\right)^{*}\right]^{2}}{2 \sigma_{m_{j}}^{2}}\right] \mathrm{d} m_{j k}
$$

where $m_{j k}$ is a generic measure related to the method, $\alpha_{j}$, and $\left(m_{j}\right)^{*}, \sigma_{m_{j}}^{2}, \sigma_{m_{j}}$, are the expected value, the variance, the rms error, respectively, of the distribution. Expected values and rms error estimators are known to be the arithmetic mean and the standard deviation, respectively, and the results of an earlier study [3] well apply to each method, $\alpha_{j}$, taken separately.

Let $f(\hat{m}) \mathrm{d} \hat{m}$ be the distribution related to the whole set of the above mentioned measure methods and statistical system, where the occurrence of the event, $E$, has been designed by the value of the random variable, $\hat{m}$, which is a linear combination of the above mentioned random variables, for sake of simplicity denoted hereafter as $m_{i}$, via the coefficients, $\omega_{i}^{*}, 1 \leq i \leq n, n=n_{1}+n_{2}+\cdots+n_{n^{\prime}}$. Owing to a theorem of statistics (e.g., [5], Chapter 8; [6], Chapter 2), the explicit expression reads:

$$
\begin{gathered}
f(\hat{m}) \mathrm{d} \hat{m}=\frac{1}{\sqrt{2 \pi} \sigma_{\hat{m}}} \exp \left\{-\frac{\left[\hat{m}-(\hat{m})^{*}\right]^{2}}{2 \sigma_{\hat{m}}^{2}}\right\} \mathrm{d} \hat{m} \\
\hat{m}=\sum_{p=1}^{n} \omega_{p}^{*} m_{p} \\
\sigma_{\hat{m}}^{2}=\sum_{p=1}^{n}\left(\omega_{p}^{*}\right)^{2} \sigma_{m_{p}}^{2}
\end{gathered}
$$

where $\hat{m}$ is related to a generic $m_{p}$ collection and $(\hat{m})^{*}, \sigma_{\hat{m}}^{2}, \sigma_{\hat{m}}$, are the expected value, the variance, the rms error, respectively, of the distribution.

The additional condition on the coefficients:

$$
\sum_{p=1}^{n} \omega_{p}^{*}=1
$$

ensures that in the special case of distributions with equal expected values, which is the one under consideration, 
$m_{1}^{*}=m_{2}^{*}=\cdots=m_{n}^{*}=m^{*},(\hat{m})^{*}=m^{*}$ via Equation (3), using a theorem of statistics (e.g., [5], Chapter 8; [6], Chapter 2).

The further condition that the variance, defined by Equation (4), has to be minimum for fixed data, implies for the coefficients, $\omega_{i}^{*}$, the following expression (e.g., [6], Chapter 4):

$$
\omega_{i}^{*}=\frac{1 / \sigma_{m_{i}}^{2}}{\sum_{q=1}^{n} 1 / \sigma_{m_{q}}^{2}}, \quad 1 \leq i \leq n
$$

and Equations (2), (3), (4), (6), reduce to:

$$
\begin{gathered}
f(\tilde{m}) \mathrm{d} \tilde{m}=\frac{1}{\sqrt{2 \pi} \sigma_{\tilde{m}}} \exp \left\{-\frac{\left(\tilde{m}-m^{*}\right)^{2}}{2 \sigma_{\tilde{m}}^{2}}\right\} \mathrm{d} \tilde{m} \\
\tilde{m}=\sum_{i=1}^{n} \omega_{p}^{*} m_{i} \\
\sigma_{\tilde{m}}^{2}=\left[\sum_{i=1}^{n} \frac{1}{\sigma_{m_{i}}^{2}}\right]^{-1} \\
\omega_{i}^{*}=\frac{\sigma_{\tilde{m}}^{2}}{\sigma_{m_{i}}^{2}}, \quad 1 \leq i \leq n
\end{gathered}
$$

where $\tilde{m}$ is a random variable and $m^{*}=(\tilde{m})^{*}, \sigma_{\tilde{m}}^{2}, \sigma_{\tilde{m}}$, are the expected value, the variance, the rms error, respectively, of the distribution.

Expected value and rms error estimators are known to be the weighted mean, $\tilde{m}$, expressed by Equation (8) where $m_{i}$ are measured values, and the standard deviation, $\tilde{\chi}$, respectively, which reads:

$$
\begin{gathered}
\tilde{\chi}=\left[\frac{1}{n-1} \sum_{i=1}^{n} \omega_{i}^{*} \tilde{\lambda}_{i}^{2}\right]^{1 / 2}, \quad 0 \leq \tilde{\chi}<+\infty \\
\tilde{\lambda}_{i}=m_{i}-\tilde{m}, \quad \sum_{i=1}^{n} \omega_{i}^{*} \tilde{\lambda}_{i}=0
\end{gathered}
$$

where $\tilde{\lambda}_{i}=m_{i}-\tilde{m}$ is the deviation from the weighted mean. It is worth emphasizing the tilde over $\chi$ and $\lambda$ means the rms error and the deviation relate to the weighted mean: neither $\tilde{\chi}$ nor $\tilde{\lambda}$, in itself, is a weighted mean. In addition, the following relations hold:

$$
\operatorname{Plim}_{n \rightarrow+\infty} \tilde{m}=m^{*}, \quad \operatorname{Plim}_{n \rightarrow+\infty} \tilde{\chi}=0, \quad \operatorname{Plim}_{n_{j} \rightarrow+\infty} \chi_{m_{j}}=\sigma_{m_{j}}
$$

where Plim has to be intended in statistical sense, according to Bernoulli's theorem (e.g., [5], Chapter 2, § 13; [6], Chapter 2); [7], Chapter 3, §3) and $\chi_{m_{j}}$ is the standard deviation inferred from data related to the measure method, $\alpha_{j}, 1 \leq j \leq n^{\prime}$ (e.g., [3]).

The substitution of Equation (8) into (12) yields the explicit expression of the deviation in terms of the measures, $m_{1}, m_{2}, \cdots, m_{n}$, as:

$$
\tilde{\lambda}_{i}=\left(1-\omega_{i}^{*}\right) m_{i}-\sum_{p=1}^{n}\left(1-\delta_{p i}\right) \omega_{p}^{*} m_{p}
$$

where $\delta_{p i}$ is the Kronecker symbol.

Using a theorem of statistics (e.g., [5], Chapter 8; [6], Chapter 2), the deviation distribution reads:

$$
f_{i}\left(\tilde{\lambda}_{i}\right) \mathrm{d} \tilde{\lambda}_{i}=\frac{1}{\sqrt{2 \pi} \sigma_{\tilde{\lambda}_{i}}} \exp \left(-\frac{1}{2} \frac{\tilde{\lambda}_{i}^{2}}{\sigma_{\tilde{\lambda}_{i}}^{2}}\right) \mathrm{d} \tilde{\lambda}_{i}
$$




$$
\sigma_{\tilde{\lambda}_{i}}^{2}=\left(1-\omega_{i}^{*}\right) \sigma_{m_{i}}^{2}=\frac{1-\omega_{i}^{*}}{\omega_{i}^{*}} \sigma_{\tilde{m}}^{2}, \quad 1 \leq i \leq n
$$

by use of Equation (10).

With regard to Equation (11), the weighted mean standard deviation distribution reads:

$$
f(\tilde{\chi}) \mathrm{d} \tilde{\chi}=\tilde{C}_{n} \iint \cdots \int_{\tilde{D}_{n}} \prod_{i=1}^{n} f_{i}\left(\tilde{\lambda}_{i}\right) \mathrm{d} \tilde{\lambda}_{i}
$$

where the random variables, $\tilde{\lambda}_{i}, 1 \leq i \leq n$, are no longer independent via Equation (12), $\tilde{C}_{n}$ is a normalizing constant and the integration domain, $\tilde{D}_{n}$, is made of the whole amount of $n$-tuples, $\left\{\tilde{\lambda}_{1}, \tilde{\lambda}_{2}, \cdots, \tilde{\lambda}_{n}\right\}$ which, via Equations (11), (12), define an interval, centered on $\tilde{\chi}$, of infinitesimal amplitude equal to $\mathrm{d} \tilde{\chi}$.

The deviations, $\tilde{\lambda}_{i}$, are dependent random variables owing to Equation (12). Conversely, any deviation is a function of the measures, $m_{1}, m_{2}, \cdots, m_{n}$, as shown by Equation (14). Accordingly, the weighted mean standard deviation, $\tilde{\chi}$, has to be expressed in terms of independent random variables.

Aiming to calculate the multiple integral on the right-hand side of Equation (17), three steps shall be performed as in the parent paper [3], where the weighted mean, $\tilde{m}$, the weighted mean rms error, $\sigma_{\tilde{m}}$, the weighted mean standard deviation, $\tilde{\chi}$, the weighted mean standard deviation distribution, $f(\tilde{\chi}) \mathrm{d} \tilde{\chi}$, are to be considered in place of their counterparts related to the arithmetic mean.

In dealing with the geometrical framework, for sake of simplicity, the formalism has to be specified as in the parent paper [3], to which an interested reader is addressed. The extension of useful formulation of analytic geometry to $(n+1)$-spaces, which shall be needed in the following, is outlined in Appendix A.

\section{Expression of $\tilde{\chi}$ in Terms of $x_{1}, x_{2}, \cdots, x_{n}$ and Related Geometrical Framework}

The generic deviation, $\tilde{\lambda}_{i}$, in terms of the errors, $x_{1}, x_{2}, \cdots, x_{n}$, can be expressed as:

$$
\begin{gathered}
\tilde{\lambda}_{i}=x_{i}-x_{\tilde{m}} \\
x_{i}=m_{i}-m^{*} \\
x_{\tilde{m}}=\tilde{m}-m^{*}=\sum_{i=1}^{n} \omega_{i}^{*} x_{i}=\tilde{x}
\end{gathered}
$$

according to the general definition of error. It is apparent the error of the weighted mean equals the weighted mean of the errors. The substitution of Equation (20) into (18) yields:

$$
\tilde{\lambda}_{i}=x_{i}-\tilde{x}, \quad \sum_{i=1}^{n} \omega_{i}^{*} x_{i}-\tilde{x}=0
$$

which shows the deviation of a measure from the weighted mean of the measures equals the deviation of the related error from the weighted mean of the errors. The right-hand side relation appearing in Equation (21) represents a $n$-plane passing through the origin within a $(n+1)$-space described by the reference frame, $\left(O x_{1} x_{2} \cdots x_{n} \tilde{x}\right)$.

The substitution of Equation (21) into (11) after some algebra yields:

$$
\frac{\sum_{i=1}^{n} \omega_{i}^{*} x_{i}^{2}}{(n-1) \tilde{\chi}^{2}}-\frac{\tilde{\chi}^{2}}{(n-1) \tilde{\chi}^{2}}=1, \quad n>1
$$

which represents a one-sheet $n$-hyperboloid where the polar axis coincides with the coordinate axis, $\tilde{x}$, the equatorial semiaxes and the polar semiaxis read:

$$
\tilde{\rho}_{i}=\sqrt{(n-1) / \omega_{i}^{*}} \tilde{\chi} ; \quad 1 \leq i \leq n, \quad \tilde{\rho}=\sqrt{n-1} \tilde{\chi}
$$


respectively ${ }^{1}$, and the equator is the intersection between the $n$-hyperboloid and the principal $n$-plane, $\left(O x_{1} X_{2} \cdots x_{n}\right)$.

The asymptotes of the $n$-hyperboloid are generatrixes of a $(n+1)$-cone where the axis coincides with the coordinate axis, $\tilde{x}$, the vertex coincides with the origin, $O$, and the lateral $n$-surface reads:

$$
\frac{\sum_{i=1}^{n} \omega_{i}^{*} x_{i}^{2}}{(n-1) \tilde{\chi}^{2}}-\frac{\tilde{x}^{2}}{(n-1) \tilde{\chi}^{2}}=0, \quad n>1
$$

which may be considered as the equation of the $(n+1)$-cone.

The generatrixes lying on the principal plane, $\left(O x_{i} \tilde{x}\right)$, are expressed as:

$$
g_{i}: \frac{\sqrt{\omega_{i}^{*}} x_{i}}{\sqrt{n-1} \tilde{\chi}} \mp \frac{\tilde{x}}{\sqrt{n-1} \tilde{\chi}}=0 ; \quad n>1, \quad 1 \leq i \leq n
$$

which can be extended to a generic direction, $\left(\sqrt{\omega_{1}^{*}} x_{1}, \sqrt{\omega_{2}^{*}} x_{2}, \cdots, \sqrt{\omega_{n}^{*}} x_{n}\right)$, by replacing $\sqrt{\omega_{i}^{*}} x_{i}$ with $x=\sqrt{\omega_{1}^{*} x_{1}^{2}+\omega_{2}^{*} x_{2}^{2}+\cdots+\omega_{n}^{*} x_{n}^{2}}$.

Using general formulation of analytic geometry extended to $(n+1)$-spaces, Equations (108) and (111), it can be ascertained if the angle, $\alpha$, formed by the coordinate axis, $x$, and the $n$-plane, expressed by Equation (21), lies between the minimum and the maximum angle, $\beta_{\min }, \beta_{\max }$, formed by the coordinate axis, $\tilde{x}$, and related generatrixes of the $(n+1)$-cone, expressed by Equation (25). To this respect, a necessary and sufficient condition is that the $n$-plane, expressed by Equation (21), is tangent to the $(n+1)$-cone, expressed by Equation (24), along a generatrix, $g_{t}$, which can be determined via the condition that the generic generatrix, $g$, lies on the $n$-plane, expressed by Equation (21).

Keeping in mind the $(n+1)$-cone has vertex on the origin and axis coinciding with the coordinate axis, $\tilde{x}$, the equation of the generic generatrix reads:

$$
g: \frac{x_{1}}{\ell_{1}}=\frac{x_{2}}{\ell_{2}}=\cdots=\frac{x_{n}}{\ell_{n}}=\frac{\tilde{x}}{\tilde{\ell}}
$$

which implies $x_{i}=\left(\ell_{i} / \tilde{\ell}\right) \tilde{x}$. Accordingly, Equation (24) reduces to:

$$
\sum_{i=1}^{n} \omega_{i}^{*}\left(\frac{\ell_{i}}{\tilde{\ell}} \tilde{x}\right)^{2}-\tilde{x}^{2}=0
$$

that is equivalent to:

$$
\tilde{\ell}^{2}=\sum_{i=1}^{n} \omega_{i}^{*} \ell_{i}^{2}
$$

where, in the case under discussion of generatrixes, the square coefficient, $\tilde{\ell}^{2}$, equals the weighted mean of the square coefficients, $\ell_{1}^{2}, \ell_{2}^{2}, \cdots, \ell_{n}^{2}$. Finally, the substitution of Equation (28) into (26) yields:

$$
g: \frac{x_{1}}{\ell_{1}}=\frac{x_{2}}{\ell_{2}}=\cdots=\frac{x_{n}}{\ell_{n}}=\frac{\tilde{x}}{\left[\sum_{i=1}^{n} \omega_{i}^{*} \ell_{i}^{2}\right]^{1 / 2}}
$$

and the condition of parallelism between $g$ and the $n$-plane, expressed by Equation (21), selects a special generatrix, $g=g_{t}$, where the above mentioned $n$-plane is tangent to the $(n+1)$-cone, expressed by Equation

${ }^{1}$ In the special case of the arithmetic mean, $\omega_{i}^{*}=1 / n, \quad 1 \leq i \leq n$, Equation (23) reduces to: $\bar{\rho}_{i}=\sqrt{(n-1) n} \bar{\chi}, \quad 1 \leq i \leq n ; \quad \bar{\rho}=\sqrt{n-1} \bar{\chi}$, respectively. Then Equation (18) appearing in an earlier investigation [3] has to be related to the polar semiaxis instead of the equatorial semiaxis, see corrigendum in Appendix D. 
(24).

Owing to Equation (113), the result is:

$$
\tilde{\ell}=\sum_{i=1}^{n} \omega_{i}^{*} \ell_{i}
$$

where, in the case under discussion of the generatrix, $g_{t}$, the coefficient, $\tilde{\ell}$, equals the weighted mean of the coefficients, $\ell_{1}, \ell_{2}, \cdots, \ell_{n}$. The further condition, expressed by Equation (28), necessarily implies

$\ell_{1}=\ell_{2}=\cdots=\ell_{n}=1$, as $\ell_{i} \neq 0$ is needed to define a straight line in the $(n+1)$-space under the validity of Equations (28) and (30).

Accordingly, the generatrix of the $(n+1)$-cone, defined by Equation (24), where the $n$-plane, defined by Equation (21), is tangent, can be expressed as:

$$
g_{t}: x_{1}=x_{2}=\cdots=x_{n}=\tilde{x}
$$

which is the $(n+1)$-sector ${ }^{2}$ of the first and $2^{n+1}$ th $2^{n+1}$-ant $^{3}$ of the reference frame, $\left(O x_{1} x_{2} \cdots x_{n} \tilde{x}\right)$.

Let $g_{t p}$ be the projection of $g_{t}$ on the principal $n$-plane, $\left(O x_{1} x_{2} \cdots x_{n}\right)$. An explicit expression can be obtained erasing the additional coordinate, $\tilde{x}$, from the definition of $g_{t}$, Equation (31). The result is:

$$
g_{t p}: x_{1}=x_{2}=\cdots=x_{n}
$$

which is the $n$-sector of the first and $2^{n}$ th $2^{n}$-ant of the reference frame, $\left(O x_{1} x_{2} \cdots x_{n}\right)$.

Let $p_{0}$ be the straight $(n-1)$-line, intersection between the $n$-plane, $p$, defined by Equation (21), and the principal $n$-plane, $\tilde{x}=0$. The expression of $p_{0}$ can be obtained by erasing the additional coordinate, $\tilde{x}$, from Equation (21). The result is:

$$
p_{0}: \sum_{i=1}^{n} \omega_{i}^{*} x_{i}=0
$$

where $p_{0}$ passes through the origin, as expected.

Let $\gamma=\widehat{g_{t p} p_{0}}, \quad 0 \leq \gamma \leq \pi / 2$, be the angle formed by the straight line, $g_{t p}$, and the straight $(n-1)$-line, $p_{0}$. The particularization of Equation (107) to the case under discussion $\left(a_{1}=a_{2}=\cdots=a_{n}=1 ; \tilde{a}=0 ; \ell_{1}=\ell_{2}=\cdots=\ell_{n}=1 ; \tilde{\ell}=0\right)$ yields:

$$
\sin \gamma=\frac{1}{\left[\sum_{i=1}^{n}\left(\omega_{i}^{*}\right)^{2}\right]^{1 / 2} \sqrt{n}}
$$

which implies $g_{t p}$ is normal to $p_{0}$ if and only if $\omega_{i}^{*}=1 / n, 1 \leq i \leq n$, to avoid contradiction with Equation (5), or in other words the weighted mean reduces to the arithmetic mean.

In summary, the weighted mean standard deviation, $\tilde{\chi}$, can be expressed as a function of the errors, $x_{1}, x_{2}, \cdots, x_{n}$, and their weighted mean, $\tilde{x}$, via Equation (22), which represents a one-sheet $n$-hyperboloid where the polar axis coincides with the coordinate axis, $\tilde{x}$, and the asymptotes are the generatrixes of a $(n+1)$ cone, expressed by Equation (24), with vertex on the origin of the reference frame, $\left(O x_{1} x_{2} \cdots x_{n} \tilde{x}\right)$. The condition that the weighted mean of deviations is null, Equation (21), defines a $n$-plane, $p$, passing through the origin, which is tangent to the above mentioned $(n+1)$-cone at the generatrix, $g_{t}$, coinciding with the $(n+1)$ sector of the first and $2^{n+1}$ th $2^{n+1}$-ant, according to Equation (31). The straight $(n-1)$-line, $p_{0}$, intersection between the $n$-plane, $p$, and the principal $n$-plane, $\tilde{x}=0$, is normal to the projection, $p_{t p}$, of the generatrix, $g_{t}$, on the principal $n$-plane, $\tilde{x}=0$, if and only if the weighted mean reduces to the arithmetic mean, $\omega_{i}^{*}=1 / n, 1 \leq i \leq n$, according to Equation (34). In this limit, the results of the current section reduce to their counterparts in the parent paper [3].

\footnotetext{
${ }^{2}$ In particular, 2-sector reads bisector, 3-sector reads trisector, 4-sector reads quadrisector, and so on.

${ }^{3}$ In particular, 2-ant reads versant, 4-ant reads quadrant, 8-ant reads octant, and so on.
} 


\section{Expression of $f(\tilde{\chi}) \mathrm{d} \tilde{\chi}$ in Terms of $x_{1}, x_{2}, \cdots, x_{n}$, and Related Geometrical Framework}

According to the above results, (i) the points, $\left(x_{1}, x_{2}, \cdots, x_{n}, \tilde{x}\right)$, related to a fixed value of the weighted mean standard deviation, $\tilde{\chi}$, lie on a one-sheet $n$-hyperboloid, defined by Equation (22), and (ii) the points, $\left(x_{1}, x_{2}, \cdots, x_{n}, \tilde{x}\right)$, for which the weighted mean of deviations from the weighted mean is null, lie on a $n$-plane, defined by Equation (21). The combination of Equations (20)-(22), yields:

$$
\sum_{i=1}^{n} \omega_{i}^{*} x_{i}^{2}=\tilde{x}^{2}+\tilde{\rho}^{2}=\left[\sum_{i=1}^{n} \omega_{i}^{*} x_{i}\right]^{2}+\tilde{\rho}^{2}
$$

where the polar semiaxis of the $n$-hyperboloid, $\tilde{\rho}$, is defined by Equation (23).

In terms of the errors, $x_{1}, x_{2}, \cdots, x_{n}$, Equation (35) represents the intersection between the above mentioned $n$-hyperboloid and $n$-plane, projected on the principal plane, $\tilde{x}=0$, as:

$$
i_{p}: \sum_{i=1}^{n} \sum_{j=1}^{n} \omega_{i}^{*}\left(\delta_{i j}-\omega_{j}^{*}\right) x_{i} x_{j}=\sum_{i=1}^{n} \omega_{i}^{*}\left(1-\omega_{i}^{*}\right) x_{i}^{2}-2 \sum_{i=2}^{n} \sum_{j=1}^{i-1} \omega_{i}^{*} \omega_{j}^{*} x_{i} x_{j}=\tilde{\rho}^{2}
$$

where, with regard to the middle side, the single sum is made of $n$ square terms and the double sum of $n(n-1) / 2$ mixed products. The $(n-1)$-line, $i_{p}$, is the domain of the distribution, $f(\tilde{\chi}) \mathrm{d} \tilde{\chi}$, depending on the weighted mean standard deviation, $\tilde{\chi}$, via the errors, $x_{1}, x_{2}, \cdots, x_{n}$.

After performing some algebra, Equation (36) may be cast under the equivalent form [6], Chapter 4:

$$
\sum_{i=2}^{n} \sum_{j=1}^{i-1} \omega_{i}^{*} \omega_{j}^{*}\left(x_{i}-x_{j}\right)^{2}=\tilde{\rho}^{2}
$$

which is a $(n-1)$-quadric where the coefficients of the first-degree terms are null and the axis coincides with the $n$-sector, $g_{t p}$, defined by Equation (32). In addition, the quadratic form on the left-hand side of Equation (37) is clearly positive definite.

The canonical form of the above mentioned $(n-1)$-quadric can be attained passing from the starting reference frame, $\left(O x_{1} X_{2} \cdots x_{n}\right)$, to the resulting reference frame, $\left(O X_{1} X_{2} \cdots X_{n}\right)$, via rigid rotation around the origin, where the resulting coordinate axes, $X_{1}, X_{2}, \cdots, X_{n}$, coincide with the principal axes of the $n$-surface bounded by the $(n-1)$-quadric and, without loss of generality, $X_{1}$ may be chosen as polar axis. To this aim, the direction cosines must be determined where, in general, $\gamma_{\ell k}$ is the cosine of the angle formed by the resulting coordinate axis, $X_{\ell}$, and the starting coordinate axis, $x_{k}$, within the starting reference frame; $\Gamma_{k \ell}$ is the cosine of the angle formed by the starting coordinate axis, $x_{k}$, and the resulting coordinate axis, $X_{\ell}$, within the resulting reference frame; by definition, $\gamma_{\ell k}=\Gamma_{k \ell}, 1 \leq \ell \leq n, 1 \leq k \leq n$.

Following the procedure outlined in the parent paper [3], the direction cosines, $\gamma_{1 k}$, related to the coordinate axis, $X_{1}$, read:

$$
\gamma_{11}=\gamma_{12}=\cdots=\gamma_{1 n}=\frac{1}{\sqrt{n}}
$$

conformly to Equation (32). The remaining coordinate axes, $X_{2}, X_{3}, \cdots, X_{n}$, can be arbitrarily selected in that they are related to the $n-2$ principal axes of the $(n-2)$-ellipse, centered on the origin and normal to the coordinate axis, $X_{1}$. For this reason, the starting and the resulting reference frame are not needed to be congruent provided the Jacobian determinant is orthogonal, $J\left(X_{1}, X_{2}, \cdots, X_{n}\right)=\mp 1$.

The term, $x_{i}-x_{j}$, appearing in Equation (37), within the resulting reference frame can be expressed as:

$$
x_{i}-x_{j}=\sum_{\ell=2}^{n}\left(\Gamma_{i \ell}-\Gamma_{j \ell}\right) X_{\ell}
$$

which is independent of the coordinate, $X_{1}$, related to the polar axis of the $(n-1)$-quadric, as $\Gamma_{i 1}=\gamma_{1 i}=\gamma_{1 j}=\Gamma_{j 1}$, owing to Equation (38). The explicit expression of the table of direction cosines, $T_{n}=\left\|\Gamma_{k \ell}\right\|$, reads [6], Chapter 4: 


$$
T_{n}=\left\|\begin{array}{|lccccc}
\frac{1}{\sqrt{n}} & \frac{1}{\sqrt{2 \times 1}} & \frac{1}{\sqrt{3 \times 2}} & \cdots & \frac{1}{\sqrt{(n-1)(n-2)}} & \frac{1}{\sqrt{n(n-1)}} \\
\frac{1}{\sqrt{n}} & \frac{-1}{\sqrt{2 \times 1}} & \frac{1}{\sqrt{3 \times 2}} & \cdots & \frac{1}{\sqrt{(n-1)(n-2)}} & \frac{1}{\sqrt{n(n-1)}} \\
\frac{1}{\sqrt{n}} & 0 & \frac{-2}{\sqrt{3 \times 2}} & \cdots & \frac{1}{\sqrt{(n-1)(n-2)}} & \frac{1}{\sqrt{n(n-1)}} \\
\vdots & \vdots & \vdots & \ddots & \vdots & \vdots \\
\frac{1}{\sqrt{n}} & 0 & 0 & \cdots & \frac{-(n-2)}{\sqrt{(n-1)(n-2)}} & \frac{1}{\sqrt{n(n-1)}} \\
\frac{1}{\sqrt{n}} & 0 & 0 & \cdots & 0 & \frac{-(n-1)}{\sqrt{n(n-1)}}
\end{array}\right\|
$$

where its counterpart related to $(n-1)$-planes, $T_{n-1}$, can be obtained from $T_{n}$ along the following steps: (i) erase the $n$-th line and the $n$-th column; and (ii) change all the elements of the first column from $1 / \sqrt{n}$ to $1 / \sqrt{n-1}$.

The $(n-1)$-quadric, expressed by Equation (37), may be cast under the equivalent form:

$$
\tilde{\rho}^{2}=\sum_{i=2}^{n-1} \sum_{j=1}^{i-1} \omega_{i}^{*} \omega_{j}^{*}\left(x_{i}-x_{j}\right)^{2}+\sum_{j=1}^{n-1} \omega_{n}^{*} \omega_{j}^{*}\left(x_{n}-x_{j}\right)^{2}
$$

where the contribution related to the coordinate axis, $x_{n}$, has been written separately. The substitution of Equation (39) into (41) yields the equation of the $(n-1)$-quadric in the resulting reference frame, $\left(O X_{1} X_{2} \cdots X_{n}\right)$, as:

$$
\begin{aligned}
\tilde{\rho}^{2} & =\sum_{i=2}^{n} a_{i i}^{(n)} X_{i}^{2}+2 \sum_{i=3}^{n} \sum_{j=2}^{i-1} a_{i j}^{(n)} X_{i} X_{j} \\
& =\sum_{i=2}^{n-1} a_{i i}^{(n-1)} X_{i}^{2}+2 \sum_{i=3}^{n-1} \sum_{j=2}^{i-1} a_{i j}^{(n-1)} X_{i} X_{j}+\sum_{k=1}^{n-1} \omega_{n}^{*} \omega_{k}^{*}\left[\sum_{\ell=2}^{n}\left(\Gamma_{n \ell}-\Gamma_{k \ell}\right) X_{\ell}\right]^{2}
\end{aligned}
$$

where the coefficients, $a_{i i}^{(n)}, a_{i j}^{(n)}, 2 \leq j<i \leq n-1$, have the same formal espression as their counterparts, $a_{i i}^{(n-1)}, a_{i j}^{(n-1)}$, related to the $(n-2)$-quadric in the reference frame, $\left(O X_{1} X_{2} \cdots X_{n-1}\right)$, but different numerical value due to the presence of the coefficients, $\omega_{i}^{*}$, conditioned by Equation (5).

The coordinate, $X_{1}$, does not appear in Equation (42), which necessarily implies the $(n-1)$-quadric is the orthogonal section of a $n$-cylinder, normal to the polar axis, $X_{1}$. The presence of mixed products, $X_{i} X_{j}$, in Equation (42), necessarily implies the principal axes of the orthogonal section are not coincident with the coordinate axes, $X_{2}, X_{3}, \cdots, X_{n}$. Then a rigid rotation of the reference frame, $\left(O X_{1} X_{2} \cdots X_{n}\right)$, around the coordinate axis, $X_{1}$, is needed to make the principal axes of the $(n-1)$-ellipse coincide with the remaining coordinate axes. To this aim, three different attempts shall be exploited. In the special case where the weighted mean reduces to the arithmetic mean, the $(n-1)$-ellipse reduces to a $(n-1)$-circle and a simpler procedure can be used [3].

\subsection{First Attempt}

The explicit expression of the coefficients, $a_{i i}^{(n)}, a_{i j}^{(n)} ; a_{i i}^{(n-1)}, a_{i j}^{(n-1)}$; appearing in Equation (42), may be determined along the following steps [6], Chapter 4.

- Particularize Equations (39) and (40) to the special case, $n=m+1, i=m+1$, and express the differences, $x_{m+1}-x_{j}$, in terms of direction cosines, $\Gamma_{m+1, \ell}, \quad \Gamma_{j l}$, and resulting coordinates, $X_{\ell}, 2 \leq \ell \leq m+1$. 
- Express the square differences, $\left(x_{m+1}-x_{j}\right)^{2}$, by writing the terms in $m+1$ separately from the remaining ones.

- Substitute the above expressions into the second term on the right-hand side of Equation (41) particularized to the case under discussion, $n=m+1$.

- Substitute the result into the right-hand side of Equation (42) particularized to the case under discussion, $n=m+1$, and compare with the middle side, term by term in $X_{i}^{2}$ and $X_{i} X_{j}$.

The result is [6], Chapter 4:

$$
\begin{gathered}
a_{i i}^{(m+1)}=a_{i i}^{(m)}+\frac{\omega_{m+1}^{*}}{i(i-1)}\left[\sum_{k=1}^{i-1} \omega_{k}^{*}+(i-1)^{2} \omega_{i}^{*}\right] ; \quad 2 \leq i \leq m \\
a_{m+1, m+1}^{(m+1)}=\frac{(m+1)^{2} \omega_{m+1}^{*} \sum_{k=1}^{m} \omega_{k}^{*}}{(m+1) m} \\
a_{i j}^{(m+1)}=a_{i j}^{(m)}-\frac{\omega_{m+1}^{*}}{\sqrt{i(i-1)}} \frac{(j-1) \omega_{j}^{*}}{\sqrt{j(j-1)}}+\sum_{k=1}^{j-1} \frac{\omega_{k}^{*}}{\sqrt{i(i-1)}} \frac{\omega_{m+1}^{*}}{\sqrt{j(j-1)}} ; \quad 2 \leq j<i \leq m \\
a_{m+1, j}^{(m+1)}=\frac{(m+1) \omega_{m+1}^{*}}{(m+1) m}+\frac{\sum_{k=1}^{j-1} \omega_{k}^{*}-(j-1) \omega_{j}^{*}}{\sqrt{j(j-1)}}
\end{gathered}
$$

and the recursive application of Equations (43a) and (43c), with due account taken of (43b) and (43d), after some algebra yields:

$$
\begin{array}{ll}
a_{i i}^{(m+1)}=\frac{1}{i(i-1)}\left[i^{2} \omega_{i}^{*} \sum_{k=1}^{i-1} \omega_{k}^{*}+\sum_{\ell=i+1}^{m+1} \omega_{\ell}^{*}(i-1)^{2} \omega_{i}^{*}+\sum_{\ell=i+1}^{m+1} \omega_{\ell}^{*} \sum_{k=1}^{i-1} \omega_{k}^{*}\right] ; & 2 \leq i \leq m+1 \\
a_{i j}^{(m+1)}=\frac{1}{\sqrt{i(i-1)}} \frac{1}{\sqrt{j(j-1)}}\left[i \omega_{i}^{*}+\sum_{\ell=i+1}^{m+1} \omega_{\ell}^{*}\right]\left[\sum_{k=1}^{j-1} \omega_{k}^{*}-(j-1) \omega_{j}^{*}\right] ; \quad 2 \leq j<i \leq m+1
\end{array}
$$

with regard to $(m+1)$-spaces, and:

$$
\begin{gathered}
a_{i i}^{(m)}=\frac{1}{i(i-1)}\left[i^{2} \omega_{i}^{*} \sum_{k=1}^{i-1} \omega_{k}^{*}+\sum_{\ell=i+1}^{m+1} \omega_{\ell}^{*}(i-1)^{2} \omega_{i}^{*}+\sum_{\ell=i+1}^{m} \omega_{\ell}^{*} \sum_{k=1}^{i-1} \omega_{k}^{*}\right] ; \quad 2 \leq i \leq m \\
a_{i j}^{(m)}=\frac{1}{\sqrt{i(i-1)}} \frac{1}{\sqrt{j(j-1)}}\left[i \omega_{i}^{*}+\sum_{\ell=i+1}^{m} \omega_{\ell}^{*}\right]\left[\sum_{k=1}^{j-1} \omega_{k}^{*}-(j-1) \omega_{j}^{*}\right] ; \quad 2 \leq j<i \leq m
\end{gathered}
$$

with regard to $m$-planes.

Finally, the symmetry relations:

$$
a_{i j}^{(n)}=a_{j i}^{(n)}, \quad n=m, m+1, \quad 2 \leq j<i \leq n
$$

are due to the appearence of related coefficients in the expression of a quadratic form on the middle side of Equation (42).

With regard to the $(n-1)$-quadric, expressed by Equation (42), the above results do not allow finding a table of direction cosines, counterpart of Equation (40), related to a rigid rotation of the reference frame,

$\left(O X_{1} X_{2} \cdots X_{n}\right)$, around the coordinate axis, $X_{1}$, yielding a new reference frame where the $(n-1)$-quadric is expressed in canonical form. To this aim, general transformations of the reference frame have to be considered, including orthogonal transformations as special cases. That is the case of linear transformations, which imply matrix operations (e.g., [8], Tome III, Part I, Chapter II).

\subsection{Second Attempt}

With regard to the $(n-1)$-quadric, expressed by Equation (36), the matrix of the quadratic form on the left- 
hand side reads:

$$
\Omega=\left\|\begin{array}{cccc}
\omega_{1}^{*}\left(1-\omega_{1}^{*}\right) & -\omega_{1}^{*} \omega_{2}^{*} & \cdots & -\omega_{1}^{*} \omega_{n}^{*} \\
-\omega_{2}^{*} \omega_{1}^{*} & \omega_{2}^{*}\left(1-\omega_{2}^{*}\right) & \cdots & -\omega_{2}^{*} \omega_{n}^{*} \\
\vdots & \vdots & \ddots & \vdots \\
-\omega_{n}^{*} \omega_{1}^{*} & -\omega_{n}^{*} \omega_{2}^{*} & \cdots & \omega_{n}^{*}\left(1-\omega_{n}^{*}\right) \|
\end{array}\right\|
$$

where the determinant, $\operatorname{det}(\Omega)$, is the discriminant of the quadratic form, and the elements are real numbers in the case under consideration. Due to the symmetry of the elements of quadratic forms, $\omega_{i}^{*}\left(\delta_{i j}-\omega_{j}^{*}\right)=\omega_{j}^{*}\left(\delta_{j i}-\omega_{i}^{*}\right)$ concerning Equation (36), the matrix of the quadratic form coincides with the transpose matrix, $\Omega=\tilde{\Omega}$.

Let $A$ be a matrix of order, $n$, related to an assigned linear transformation from a starting reference frame, $\left(O x_{1} X_{2} \cdots x_{n}\right)$, to a resulting reference frame, $\left(O X_{1} X_{2} \cdots X_{n}\right)$, as:

$$
\left(X_{1}, X_{2}, \cdots, X_{n}\right)=A\left(x_{1}, x_{2}, \cdots, x_{n}\right)
$$

where the coordinates define the same vector in the appropriate reference frame.

The linear transformation reduces to an orthogonal transformation if and only if the elements of the matrix, $A$, satisfy the orthogonality conditions (e.g., [8], Tome III, Part I, Chapter II):

$$
\sum_{\ell=1}^{n} a_{\ell i} a_{\ell j}=\delta_{i j}, \quad \sum_{k=1}^{n} a_{i k} a_{j k}=\delta_{i j}
$$

concerning lines and columns, respectively.

According to the theory of linear transformations, the expression of the quadratic form on the left-hand side of Equation (36), in canonical form, takes place via the determination of an orthogonal matrix, $A$, which makes the product matrix, $C=A^{-1} \Omega A, A^{-1}$ inverse matrix of $A$, be diagonal. Then $C$ is the matrix of the linear transformation of interest. The above procedure implies the solution of an eigenvalue problem (e.g., [8], Tome III, Part I, Chapter II).

With regard to the $(n-1)$-quadric, expressed by Equation (36), the eigenvalue equation is:

$$
0=\phi(\lambda)=\operatorname{det}(\Omega-\Lambda)=\left|\begin{array}{cccc}
\omega_{1}^{*}\left(1-\omega_{1}^{*}\right)-\lambda & -\omega_{1}^{*} \omega_{2}^{*} & \cdots & -\omega_{1}^{*} \omega_{n}^{*} \\
-\omega_{2}^{*} \omega_{1}^{*} & \omega_{2}^{*}\left(1-\omega_{2}^{*}\right)-\lambda & \cdots & -\omega_{2}^{*} \omega_{n}^{*} \\
\vdots & \vdots & \ddots & \vdots \\
-\omega_{n}^{*} \omega_{1}^{*} & -\omega_{n}^{*} \omega_{2}^{*} & \cdots & \omega_{n}^{*}\left(1-\omega_{n}^{*}\right)-\lambda
\end{array}\right|
$$

where the matrix, $\Omega$, is defined by Equation (47); the matrix, $\Lambda$, reads $\Lambda=\lambda I, I$ unit matrix of order, $n$; and $\lambda$ is the variable. After a lot of determinant algebra, Equation (50) may be cast under the explicit form [6], Chapter 4:

$$
\begin{gathered}
\lambda \sum_{\ell=1}^{n}\left(\frac{\lambda}{\omega_{1}^{*}}-1\right) \ldots\left(\frac{\lambda}{\omega_{\ell-1}^{*}}-1\right)\left(\frac{\lambda}{\omega_{\ell+1}^{*}}-1\right) \cdots\left(\frac{\lambda}{\omega_{n}^{*}}-1\right)=0 \\
\omega_{0}^{*}=\omega_{n+1}^{*}=\frac{\lambda}{2} ; \quad \ell=1, n
\end{gathered}
$$

which is an algebraic equation of degree, $n$, as [6], Chapter 4 :

$$
(-1)^{n} \alpha_{0}^{(n)} \lambda^{n}+(-1)^{n-1} \alpha_{1}^{(n)} \lambda^{n-1}+\cdots+(-1)^{1} \alpha_{n-1}^{(n)} \lambda+(-1)^{0} \alpha_{n}^{(n)}=0
$$

where the coefficients, regardless of an inessential multiplicative constant, may be expressed as [6], Chapter 4 :

$$
\alpha_{k}^{(n)}=(k+1) \sum_{i_{1}=k+1 i_{2}=k}^{n} \sum_{i_{1}-1}^{i_{1}} \cdots \sum_{i_{k+1}=1}^{i_{k}-1} \omega_{i_{1}}^{*} \omega_{i_{2}}^{*} \cdots \omega_{i_{k+1}}^{*} ; \quad i_{1}>i_{2}>\cdots>i_{k+1} ; 0 \leq k<n
$$

and, in particular: 


$$
\alpha_{0}^{(n)}=1 ; \quad \alpha_{n}^{(n)}=\operatorname{det}(\Omega)=0
$$

where the last relation comes from Equation (47) after summation of all lines or columns on a selected line or column which, via Equation (5), yields a line or column made of null elements implying, in turn, a null determinant.

With regard to Equation (51a), the existence of $n$ solutions (eigenvalues) is ensured by the fundamental theorem of algebra, where all the solutions are real in the case under discussion, as linear orthogonal transformations are involved. In particular, the appearence of a null solution, let it be $\lambda_{n}=0$, implies the $(n-1)$ quadric, expressed by Equation (36), is degenerate to the lateral $(n-1)$-surface of a $n$-cylinder.

According to the general theory (e.g., [8], Tome III, Part I, Chapter II; [9], Vol. I, Chapter 10), a generic quadratic form where the coefficients are real, as:

$$
\phi\left(x_{1}, x_{2}, \cdots, x_{n}\right)=\sum_{i=1}^{n} \sum_{j=1}^{n} a_{i j} x_{i} x_{j} ; \quad a_{i j}=a_{j i}
$$

can be reduced to the canonical form:

$$
\phi\left(X_{1}, X_{2}, \cdots, X_{n}\right)=\sum_{i=1}^{n} \lambda_{i} X_{i}^{2}
$$

by use of a convenient linear orthogonal transformation, where $\lambda_{1}, \lambda_{2}, \cdots, \lambda_{n}$, are the eigenvalues of the matrix of the quadratic form.

In the case under discussion, with regard to Equations (23), (47), (51a), Equation (56) reduces to:

$$
\sum_{i=1}^{n-1} \lambda_{i} X_{i}^{2}=\tilde{\rho}^{2}=(n-1) \tilde{\chi}^{2}
$$

which defines a $(n-1)$-ellipse, orthogonal section of a $n$-cylinder, where the coordinate axes, $X_{1}, X_{2}, \cdots, X_{n-1}$, coincide with the principal axes of the $(n-1)$-ellipse, and the coordinate axis, $X_{n}$, coincides with the axis of the $n$-cylinder ${ }^{4}$.

The semiaxes of the $(n-1)$-ellipse are:

$$
\begin{gathered}
\tilde{a}_{i}=\left(\frac{n-1}{\lambda_{i}}\right)^{1 / 2} \tilde{\chi} ; \quad 1 \leq i \leq n-1 \\
\tilde{a}_{1}>\tilde{a}_{2}>\cdots>\tilde{a}_{n-1} ; \quad 0<\lambda_{1}<\lambda_{2}<\cdots<\lambda_{n-1}
\end{gathered}
$$

accordingly, the features of the $(n-1)$-ellipse depend on the eigenvalues only. In particular, the axis ratios, $\tilde{\epsilon}_{r s}=\tilde{a}_{r} / \tilde{a}_{s}, 1 \leq r \leq s \leq n-1$, depend on the eigenvalues only, which implies different $(n-1)$-ellipses, defined by Equation (57), are homotetic.

It is apparent from Equation (51a) that the determination of eigenvalues is not an easy matter in the general case, $n \gg 1$. For this reason, a different attempt has to be exploited, where a canonical expression of the quadratic form is inferred regardless of the eigenvalues. To this aim, general linear transformations shall be used, which reduce to orthogonal linear transformations as a special case.

\subsection{Third Attempt}

With regard to the quadratic form, expressed by Equation (55), let $n$ real linear forms be considered, among which $k_{r}$ are linearly independent, $1 \leq k_{r} \leq n$, as:

$$
Y_{m}=\sum_{\ell=1}^{n} d_{m \ell} x_{\ell} ; \quad 1 \leq m \leq n
$$

where $d_{m \ell}, 1 \leq \ell \leq n$, are coefficients satisfying the relation:

$$
\phi\left(x_{1}, x_{2}, \cdots, x_{n}\right)=\sum_{m=1}^{n} c_{m} Y_{m}^{2}
$$

${ }^{4}$ For sake of convenience, the polar axis here is denoted as $X_{n}$ instead of $X_{1}$ as done earlier in the current section. 
where, in turn, $c_{m}, 1 \leq m \leq n$, are coefficients characterized by the following properties.

(1) The number of positive, $k^{(+)}$, and negative, $k^{(-)}$, coefficients, equals the rank of the matrix of the quadratic form, $k^{(+)}+k^{(-)}=k_{r}$.

(2) With regard to the whole set of linear transformations, defined by Equation (60), the number of negative coefficients, $k^{(-)}$, positive coefficients, $k^{(+)}$, and null coefficients, $k^{(0)}=n-k^{(-)}-k^{(+)}$, remains unchanged for any element of the set, according to the theorem of inertia of quadratic forms (e.g., [8], Tome III, Part I, Chapter II; [9], Vol. I, Chapter 10).

Further considerations shall be restricted to linear transformations defined by Jacobi formulae. For additional details, an interested reader is addressed to Appendix B. Using the Jacobi formulae, Equation (60) reduces to:

$$
\phi\left(x_{1}, x_{2}, \cdots, x_{n}\right)=\sum_{k=1}^{k_{r}} \frac{Y_{k}^{2}}{A_{12 \cdots k}^{(n)} A_{12 \cdots k-1}^{(n)}}
$$

which is valid in general.

In the case of interest, $A=\Omega$, the coefficients of the quadratic form, defined by Equation (55), take the explicit form:

$$
a_{i j}=\omega_{i}^{*}\left(\delta_{i j}-\omega_{j}^{*}\right)
$$

according to Equation (47), and Equation (115) reduces to (47). Finally, Equation (114) reads:

$$
\Xi_{i}=\sum_{k=1}^{n} \omega_{i}^{*}\left(\delta_{i k}-\omega_{k}^{*}\right) x_{k} ; \quad 1 \leq i \leq n-1
$$

and Equations (116) take the explicit form [6], Chapter 4:

$$
\begin{gathered}
A_{12 \cdots k}^{(n)}=\Omega_{12 \cdots k}^{(n)}=\omega_{1}^{*} \omega_{2}^{*} \cdots \omega_{k}^{*} \sum_{\ell=k+1}^{n} \omega_{\ell}^{*} ; \quad 1 \leq i \leq n \\
A^{(n)}=\Omega^{(n)}=1 ; \quad k=0
\end{gathered}
$$

in addition, Equation (64a) may be expressed via a recursive formula, as:

$$
\begin{gathered}
\Omega_{12 \cdots k}^{(n)}=\omega_{k}^{*}\left[\Omega_{12 \cdots k-1}^{(n)}-\omega_{1}^{*} \omega_{2}^{*} \cdots \omega_{k}^{*}\right] \\
\Omega^{(n)}>\Omega_{1}^{(n)}>\cdots>\Omega_{12 \cdots n-1}^{(n)}
\end{gathered}
$$

where inequalities are due to the constraints, $0<\omega_{k}^{*}<1,1 \leq k \leq n$, conformly to Equation (5).

Let $\left(O Y_{1} Y_{2} \cdots Y_{n}\right)$ be a reference frame where the quadratic form on the left-hand side of Equation (36) can be expressed as a linear combination of pure quadratic terms, i.e. the coefficients of mixed products are null. The particularization of Equation (117) to the case under discussion yields:

$$
Y_{k}=\left|\begin{array}{ccccc}
\omega_{1}^{*}\left(1-\omega_{1}^{*}\right) & -\omega_{1}^{*} \omega_{2}^{*} & \cdots & -\omega_{1}^{*} \omega_{k-1}^{*} \omega_{1}^{*}\left(\delta_{1 k}-\omega_{k}^{*}\right) x_{k} \\
-\omega_{2}^{*} \omega_{1}^{*} & \omega_{1}^{*}\left(1-\omega_{1}^{*}\right) & \cdots & -\omega_{2}^{*} \omega_{k-1}^{*} & \Xi_{2} \\
\vdots & \vdots & \ddots & \vdots & \vdots \\
-\omega_{k-1}^{*} \omega_{1}^{*} & -\omega_{k-1}^{*} \omega_{2}^{*} & \cdots & \omega_{k-1}^{*}\left(1-\omega_{k-1}^{*}\right) & \Xi_{k-1} \\
-\omega_{k}^{*} \omega_{1}^{*} & -\omega_{k}^{*} \omega_{2}^{*} & \cdots & -\omega_{k}^{*} \omega_{k-1}^{*} & \Xi_{k}
\end{array}\right| ; \quad 2 \leq k \leq n-1
$$

where the rank of the matrix, $\Omega$, defined by Equation (47), is $k_{r}=n-1$, conformly to Equations (53) and (54), which implies the appearence of $(n-1)$ independent quadratic terms, $Y_{k}^{2}, 1 \leq k \leq n-1$, in the expression of the above mentioned quadratic form.

By use of Jacobi formulae, Equations (118), the $(n-1)$-quadric, expressed by Equations (36) and (23), may be cast under the canonical form: 


$$
\frac{Y_{1}^{2}}{\Omega_{1}^{(n)} \Omega^{(n)}}+\sum_{k=2}^{n-1} \frac{Y_{k}^{2}}{\Omega_{12 \cdots k}^{(n)} \Omega_{12 \cdots k-1}^{(n)}}=\tilde{\rho}^{2}=(n-1) \tilde{\chi}^{2}
$$

which defines a $(n-1)$-ellipse, orthogonal section of a $n$-cylinder, where the coordinate axes, $Y_{1}, Y_{2}, \cdots, Y_{n-1}$, coincide with the principal axes of the $(n-1)$-ellipse, and the coordinate axis, $Y_{n}$, coincides with the axis of the $n$-cylinder.

\subsection{Explicit Expressions}

With regard to the $(n-1)$-quadric, expressed by Equations (36) and (23), the canonical form in terms of eigenvalues, Equation (57), can be equated to its counterpart inferred from Jacobi formulae, Equation (67), as:

$$
\sum_{k=1}^{n-1} \lambda_{k} X_{k}^{2}=\frac{Y_{1}^{2}}{\Omega_{1}^{(n)} \Omega^{(n)}}+\sum_{k=2}^{n-1} \frac{Y_{k}^{2}}{\Omega_{12 \cdots k}^{(n)} \Omega_{12 \cdots k-1}^{(n)}}
$$

where the resulting coordinates, $X_{k}$, depend on the starting coordinates, $x_{1}, x_{2}, \cdots, x_{n}$, via an orthogonal transformation:

$$
X_{k}=\sum_{i=1}^{n} b_{i k} x_{i} ; \quad 1 \leq k \leq n
$$

which implies a rigid rotation around the origin in a $(n+1)$-space, together with a possible change in chirality, according to the sign of the Jacobian determinant.

Let $B$ a matrix of order, $n$, whose elements are $b_{i k}, 1 \leq i \leq n, 1 \leq k \leq n$. Accordingly, the matrix of the orthogonal transformation, expressed by Equation (69), is:

$$
\Psi=B^{-1} \Omega B=\left\|\begin{array}{cccc}
\lambda_{1} & 0 & \cdots & 0 \\
0 & \lambda_{2} & \cdots & 0 \\
\vdots & \vdots & \ddots & \vdots \\
0 & 0 & \cdots & \lambda_{n}
\end{array}\right\|
$$

in terms of eigenvalues of the matrix, $\Omega$, of the quadratic form on the left-hand side of Equation (36).

The semiaxes of the $(n-1)$-ellipse, orthogonal section of the $n$-cylinder, defined by Equation (57), via Equation (67) can be expressed as:

$$
\begin{gathered}
\tilde{a}_{k}=\left[\Omega_{12 \cdots k}^{(n)} \Omega_{12 \cdots k-1}^{(n)}\right]^{1 / 2}(n-1)^{1 / 2} \tilde{\chi} ; \quad 1 \leq k \leq n-1 \\
\tilde{a}_{1}>\tilde{a}_{2}>\cdots>\tilde{a}_{n-1}
\end{gathered}
$$

where the leading principal minors, $\Omega_{12 \ldots}^{(n)}$, are positive according to Equations (65) which, in addition, implies semiaxis inequalities.

In the special case where the weighted mean reduces to the arithmetic mean, $\omega_{i}^{*}=1 / n, 1 \leq i \leq n$, conformly to Equation (6), Equations (64) reduce to:

$$
\Omega_{12 \cdots k}^{(n)}=\frac{1}{n^{k}} \frac{n-k}{n}
$$

and the substitution of Equation (72) into (71a) yields:

$$
\begin{aligned}
\bar{a}_{k} & =\left[\frac{n-k}{n^{k+1}} \frac{n-k+1}{n^{k}}\right]^{1 / 2}(n-1)^{1 / 2} \bar{\chi} \\
\lim _{n \rightarrow+\infty} \bar{a}_{1} & =\bar{\chi} ; \quad \lim _{n \rightarrow+\infty} \bar{a}_{k}=0 ; \quad 2 \leq k \leq n-1
\end{aligned}
$$

which shows the current procedure is conceptually different from its counterpart related to the arithmetic mean, where the orthogonal section of the $n$-cylinder corresponds to a $(n-1)$-circle [3].

The apparent discrepancy can be explained in the following way. Jacobi formulae are grounded on non orthogonal linear transformations which, in themselves, imply changes in metric relations. On the other hand, eigenvalue equations are grounded on orthogonal linear transformations which, in themselves, imply conserva- 
tion of metric relations.

According to the general theory of linear transformations and quadratic forms (e.g., [8], Tome III, Part I, Chapter II; [9], Vol. I, Chapter 10), the coefficients of the eigenvalue equation, expressed by Equations (51)-(52), regardless of an inessential multiplicative constant, coincide with the invariants of the matrix, $\Omega$, expressed by Equation (47), with regard to the quadratic form on the left-hand side of Equation (36). The above mentioned invariants, $\alpha_{k}^{(n)}, 0 \leq k \leq n$, remain unchanged for any matrix inferred from $\Omega$ via a similarity transformation, $U \Omega U^{-1}$, $\operatorname{det}(U) \neq 0, U$ nonsingular matrix. The matrix, $\Omega$, is symmetric in that it relates to a quadratic form where the coefficients, in the case under consideration, are real, which implies the matrix, $U$, or $B=U^{-1}$, is necessarily orthogonal.

Let $S_{x}, S_{X}$, be singular matrixes defined as:

$$
\begin{aligned}
S_{x} & =\left\|\begin{array}{cccc}
X_{1} & 0 & \cdots & 0 \\
X_{2} & 0 & \cdots & 0 \\
\vdots & \vdots & \ddots & \vdots \\
X_{n} & 0 & \cdots & 0
\end{array}\right\| \\
S_{X} & =\left\|\begin{array}{cccc}
X_{1} & 0 & \cdots & 0 \\
X_{2} & 0 & \cdots & 0 \\
\vdots & \vdots & \ddots & \vdots \\
X_{n} & 0 & \cdots & 0
\end{array}\right\|
\end{aligned}
$$

where $x_{1}, x_{2}, \cdots, x_{n}$, are starting coordinates and $X_{1}, X_{2}, \cdots, X_{n}$, are resulting coordinates of a reference frame where the matrix of the quadratic form is diagonal via an orthogonal transformation using the matrix, $B$. Accordingly, the following relations hold:

$$
S_{X}=B^{-1} S_{x} ; \quad S_{x}=B S_{X} ; \quad[\operatorname{det}(B)]^{2}=1
$$

where the elements, $b_{i j}$, of the matrix, $B$, can be determined by solving $n$ systems of equations, extracted from the condition that the matrix, $\Psi=B^{-1} \Omega B$, is diagonal, and from the knowledge of the eigenvalues.

According to the general theory of linear transformations and quadratic forms (e.g., [8], Tome III, Part I, Chapter II; [9], Vol. I, Chapter 10), the above mentioned systems of equations read:

$$
\left\{\begin{array}{c}
\left(\omega_{11}-\lambda_{k}\right) b_{1 k}+\omega_{12} b_{2 k}+\cdots+\omega_{1 n} b_{n k}=0 \\
\omega_{21} b_{1 k}+\left(\omega_{22}-\lambda_{k}\right) b_{2 k}+\cdots+\omega_{2 n} b_{n k}=0 \\
\vdots \\
\omega_{n 1} b_{1 k}+\omega_{n 2} b_{2 k}+\cdots+\left(\omega_{n n}-\lambda_{k}\right) b_{n k}=0
\end{array}\right.
$$

where $\omega_{i j}$ are elements of the matrix, $\Omega$, and $\lambda_{k}$ is the $k$-th eigenvalue on a total of $n: \lambda_{1}, \lambda_{2}, \cdots, \lambda_{n}$.

The validity of the eigenvalue equation, expressed by Equation (51), necessarily implies the determinant of the system, defined by Equation (77), is null in the case of interest or, in other words, the existence of infinite solutions. On the other hand, the condition of unit norm:

$$
\sum_{\ell=1}^{n} b_{\ell k}^{2}=1 ; \quad 1 \leq k \leq n
$$

allows the selection of a special solution (regardless of the sign) among infinite others.

The particularization of the system, defined by Equation (77), to the case of interest, $\omega_{i j}=\omega_{i}^{*}\left(\delta_{i j}-\omega_{j}^{*}\right)$, and to the eigenvalue, $\lambda_{n}=0$, reads:

$$
\left\{\begin{array}{c}
+\left[\omega_{1}^{*}-\left(\omega_{1}^{*}\right)^{2}\right] b_{1 n}-\omega_{1}^{*} \omega_{2}^{*} b_{2 n}-\cdots-\omega_{1}^{*} \omega_{n}^{*} b_{n n}=0 \\
-\omega_{2}^{*} \omega_{1}^{*} b_{1 n}+\left[\omega_{2}^{*}-\left(\omega_{2}^{*}\right)^{2}\right] b_{2 n}-\cdots-\omega_{2}^{*} \omega_{n}^{*} b_{n n}=0 \\
\vdots \\
-\omega_{n}^{*} \omega_{1}^{*} b_{1 n}-\omega_{n}^{*} \omega_{2}^{*} b_{2 n}-\cdots+\left[\omega_{n}^{*}-\left(\omega_{n}^{*}\right)^{2}\right] b_{n n}=0 .
\end{array}\right.
$$


which is equivalent to:

$$
\left\{\begin{array}{c}
b_{1 n}-\sum_{\ell=1}^{n} \omega_{\ell}^{*} b_{\ell n}=0 ; \\
b_{2 n}-\sum_{\ell=1}^{n} \omega_{\ell}^{*} b_{\ell n}=0 ; \\
\vdots \\
b_{n n}-\sum_{\ell=1}^{n} \omega_{\ell}^{*} b_{\ell n}=0 .
\end{array}\right.
$$

accordingly, the following relations hold:

$$
b_{1 n}=b_{2 n}=\cdots=b_{n n}
$$

and the substitution of Equation (81) into (78), particularized to $k=n$, yields:

$$
b_{\ell n}=\mp \frac{1}{\sqrt{n}} ; \quad 1 \leq \ell \leq n
$$

where the positive sign relates to Equation (38).

Using the definition of matrix product, Equation (76) takes the explicit form:

$$
\begin{aligned}
& X_{k}=\sum_{\ell=1}^{n} b_{\ell k} x_{\ell} ; \quad 1 \leq k \leq n \\
& x_{\ell}=\sum_{\ell=1}^{n} b_{k \ell} X_{k} ; \quad 1 \leq \ell \leq n
\end{aligned}
$$

where, in the case under discussion of orthogonal linear transformations, the inverse matrix, $B^{-1}$, is inferred from the matrix, $B$, simply by exchanging lines with columns and vice versa. With regard to the coordinate axis, $X_{n}$, the combination of Equations (82) and (83a) yields:

$$
X_{n}=\mp \sum_{\ell=1}^{n} \frac{x_{\ell}}{\sqrt{n}}
$$

which is related to the eigenvalue, $\lambda_{n}=0$, then the coordinate axis, $X_{n}$, coincides with the axis of the $n$ cylinder, defined by Equation (56).

In summary, the weighted mean standard deviation distribution can be expressed as a function of the errors, $x_{1}, x_{2}, \cdots, x_{n}$, as:

$$
f(\tilde{\chi}) \mathrm{d} \tilde{\chi}=\tilde{C}_{n} \iint \cdots \int_{\tilde{D}_{n}} f_{1}\left(x_{1}\right) f_{2}\left(x_{2}\right) \cdots f_{n}\left(x_{n}\right) \mathrm{d} x_{1} \mathrm{~d} x_{2} \cdots \mathrm{d} x_{n}
$$

where $\tilde{C}_{n}$ is a normalizing constant, $\tilde{D}_{n}$ the integration domain, expressed by Equation (36) which, turned into canonical expression, Equation (56), represents an infinitely thin $n$-cylindrical corona of infinite height, axis coinciding with the coordinate axis, $X_{n}$, orthogonal section expressed by Equation (57), that is an infinitely thin, homotetic $(n-1)$-elliptical corona whose semiaxes are defined by Equation (71).

Finally, $f_{i}\left(x_{i}\right) \mathrm{d} x_{i}, 1 \leq i \leq n$, are error distributions expressed via Equation (1) as:

$$
f_{i}\left(x_{i}\right) \mathrm{d} x_{i}=\frac{1}{\sqrt{2 \pi} \sigma_{m_{i}}} \exp \left(-\frac{x_{i}^{2}}{2 \sigma_{m_{i}}^{2}}\right) \mathrm{d} x_{i} ; \quad 1 \leq i \leq n
$$

where $x_{i}$ is the error of the generic measure, $m_{i}$, and $\sigma_{m_{i}}$ is the rms error of the related distribution.

\section{Expression of $f(\tilde{\chi}) \mathbf{d} \tilde{\chi}$ in Terms of $\tilde{\chi}, \sigma_{\tilde{m}}$, and Related Geometrical Framework}

The substitution of Equations (10) and (86) into (85), after little algebra yields: 


$$
f(\tilde{\chi}) \mathrm{d} \tilde{\chi}=\tilde{C}_{n} \iint \cdots \int_{\tilde{D}_{n}} \frac{\left(\omega_{1}^{*} \omega_{2}^{*} \cdots \omega_{n}^{*}\right)^{1 / 2}}{(2 \pi)^{n / 2} \sigma_{\tilde{m}}^{n}} \exp \left(-\frac{\sum_{i=1}^{n} \omega_{i}^{*} x_{i}^{2}}{2 \sigma_{\tilde{m}}^{2}}\right) \mathrm{d} x_{1} \mathrm{~d} x_{2} \cdots \mathrm{d} x_{n}
$$

the special case, $n=2$, is shown in Figure 1 [6], Chapter 4 .

With regard to the resulting reference frame, $\left(\mathrm{OX}_{1} X_{2} \cdots X_{n}\right)$, the substitution of Equation (83b) into (84) yields:

$$
\mp X_{n}=\frac{1}{\sqrt{n}} \sum_{\ell=1}^{n}\left(\sum_{k=1}^{n-1} b_{k \ell} X_{k}+b_{n \ell} X_{n}\right)
$$

which necessarily implies the following:

$$
\sum_{k=1}^{n-1} b_{k \ell} X_{k}=0 ; \quad b_{n \ell}=\mp \frac{1}{\sqrt{n}}
$$

conformly to Equation (82).

The weighted mean of the errors, expressed by Equation (20), by use of Equations (83b) and (89), after little algebra may be cast under the equivalent form:

$$
\sum_{\ell=1}^{n} \omega_{\ell}^{*} x_{\ell}=\tilde{x}=\mp \frac{1}{\sqrt{n}} X_{n}
$$

and the substitution of Equations (23) and (90) into (35) yields:

$$
\sum_{i=1}^{n} \omega_{i}^{*} x_{i}^{2}=\left(\sum_{i=1}^{n} \omega_{i}^{*} x_{i}\right)^{2}+(n-1) \tilde{\chi}^{2}=\frac{X_{n}^{2}}{n}+(n-1) \tilde{\chi}^{2}
$$

in terms of a single resulting coordinate, $X_{n}$.

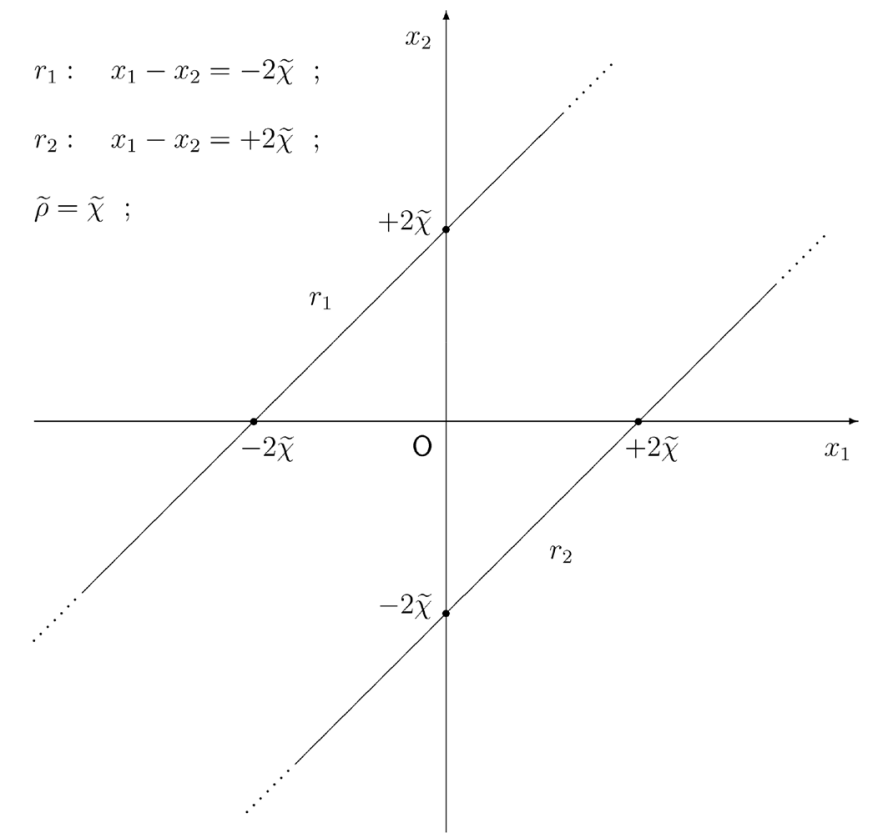

Figure 1. The integration domain, $\tilde{D}_{n}$, with regard to the reference frame, $\left(O x_{1} x_{2} \cdots x_{n}\right)$, in the special case, $n=2$. The straight lines, $r_{1}, r_{2}$, are the axes of infinitely thin bands, whose bounderies are defined by the straight lines, $r_{1}^{\mp}: x_{1}-x_{2}=-2(\tilde{\chi} \mp \mathrm{d} \tilde{\chi}) ; r_{2}^{\mp}: x_{1}-x_{2}=+2(\tilde{\chi} \mp \mathrm{d} \tilde{\chi})$. The integration domain, $\tilde{D}_{2}$, is defined by the above mentioned bands. 
The transformation, $S_{X}=B S_{X}$, appearing in Equation (76), is orthogonal with unit norm, which implies the squared Jacobian of the transformation also equals unity, $J_{n}^{2}\left(X_{1} X_{2} \cdots X_{n}\right)=1$, conformly to Equation (76). With regard to the resulting reference frame, $\left(\mathrm{OX}_{1} \mathrm{X}_{2} \cdots X_{n}\right)$, after a change of variables (e.g., [8], Chapter III, $\S 4.10$; [6], Chapter 4) by use of Equation (91) keeping in mind $J_{n}^{2}=1$, Equation (87) translates into ${ }^{5}$

$$
f(\tilde{\chi}) \mathrm{d} \tilde{\chi}=\tilde{C}_{n} \iint \cdots \int_{\tilde{\Delta}_{n}} \frac{\left(\omega_{1}^{*} \omega_{2}^{*} \cdots \omega_{n}^{*}\right)^{1 / 2}}{(2 \pi)^{n / 2} \sigma_{\tilde{m}}^{n}} \exp \left[-\frac{(n-1) \tilde{\chi}^{2}+X_{n}^{2} / n}{2 \sigma_{\tilde{m}}^{2}}\right] \mathrm{d} X_{1} \mathrm{~d} X_{2} \cdots \mathrm{d} X_{n}
$$

where the integration domain, $\tilde{\Delta}_{n}$, is an infinitely thin, homotetic $n$-cylindrical corona with axis, $X_{n}$, and semiaxes defined by Equation (71). The special case, $n=2$, is shown in Figure 2 [6], Chapter 4.

With regard to the principal $(n-1)$-plane, $\left(O X_{1} X_{2} \cdots X_{n-1}\right)$, the $(n-1)$-surface of the $(n-1)$-ellipse of semiaxes, $\tilde{a}_{k}, 1 \leq k \leq n-1$, is:

$$
\begin{gathered}
\tilde{S}_{n-1}=\frac{\left[\Gamma\left(\frac{1}{2}\right)\right]^{n-1}}{\Gamma\left(1+\frac{n-1}{2}\right)} \tilde{\epsilon}_{21} \tilde{\epsilon}_{31} \cdots \tilde{\epsilon}_{n-1,1} \tilde{a}_{1}^{n-1} \\
\tilde{\epsilon}_{\ell 1}=\frac{\tilde{a}_{\ell}}{\tilde{a}_{1}} ; \quad 2 \leq \ell \leq n-1
\end{gathered}
$$

where $\Gamma(r)$ is the Euler Gamma function, which satisfies the following relations (e.g., [10], Chapter 39, § 39.6):

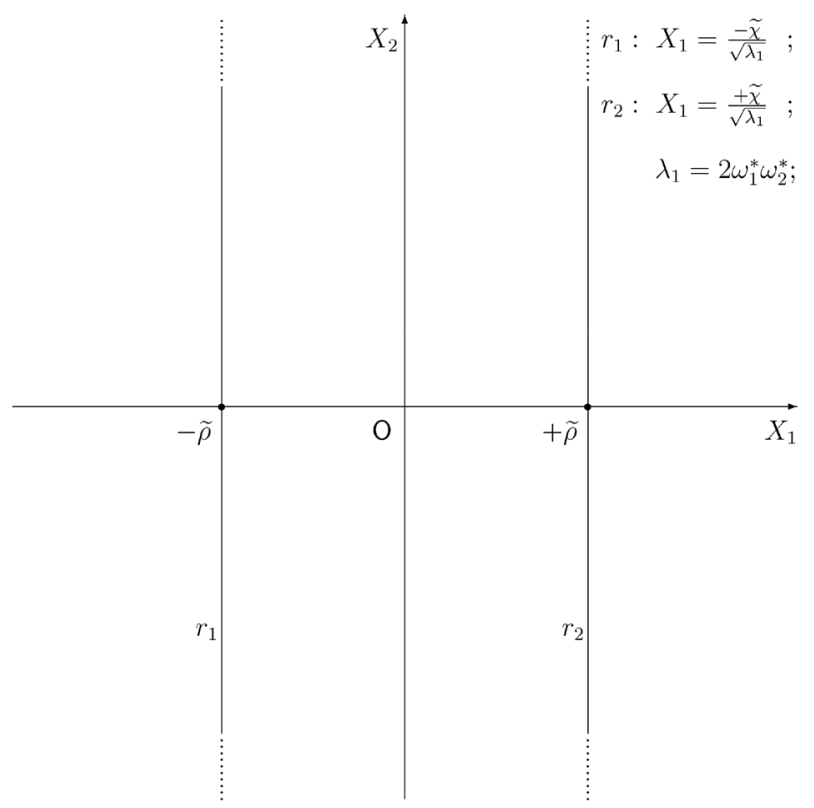

Figure 2. The integration domain, $\tilde{\Delta}_{n}$, with regard to the reference frame, $\left(O X_{1} X_{2} \cdots X_{n}\right)$, in the special case, $n=2$. The straight lines, $r_{1}, r_{2}$, are the axes of infinitely thin bands, whose boundaries are defined by the straight lines, $r_{1}^{\mp}: X_{1}=+\sqrt{2}(\tilde{\chi} \mp \mathrm{d} \tilde{\chi}) ; r_{2}^{\mp}: X_{1}=-\sqrt{2}(\tilde{\chi} \mp \mathrm{d} \tilde{\chi})$; according to Equations (51) and (57). The integration domain, $\tilde{\Delta}_{2}$, is defined by the above mentioned bands.

${ }^{5} \mathrm{~A}$ negative Jacobian determinant, $J_{n}=-1$, relates to a resulting reference frame, $\left(O X_{1} X_{2} \ldots X_{n}\right)$, which is not congruent to the starting reference frame, $\left(O x_{1} X_{2} \ldots X_{n}\right)$, and the negative sign is compensated by an even number of sign changes within the integrals appearing in Equation (87). 


$$
\begin{gathered}
\Gamma(r+1)=r \Gamma(r) ; \quad r>0 \\
\Gamma(i+1)=i ! ; \quad i=0,1,2, \cdots \\
\Gamma\left(i+\frac{1}{2}\right)=\frac{1 \times 2 \times \cdots \times(2 i-1)}{2^{i}} \sqrt{\pi} ; \quad \Gamma\left(\frac{1}{2}\right)=\sqrt{\pi} \\
\Gamma(r+1)=\sqrt{2 \pi} r^{1 / 2} r^{r} \exp (-r) F(r) \\
F(r)=\left[1+\frac{1}{12} \frac{1}{r}+\frac{1}{288} \frac{1}{r^{2}}-\frac{139}{51840} \frac{1}{r^{3}}-\cdots\right]
\end{gathered}
$$

and the particularization of Equation (93a) to the simplest cases, $n=1,2,3,4$ yields:

$$
\tilde{S}_{0}=0 ; \quad \tilde{S}_{1}=2 \tilde{a}_{1} ; \quad \tilde{S}_{2}=\pi \tilde{\epsilon}_{21} \tilde{a}_{1}^{2} ; \quad \tilde{S}_{3}=\frac{4 \pi}{3} \tilde{\epsilon}_{21} \tilde{\tau}_{31} \tilde{a}_{1}^{3}
$$

with regard to points, segments, ellipses, ellipsoids, respectively.

In particular, $n=1$ implies a single deviation from the mean, $\tilde{\lambda}_{1}=0$ conformly to Equation (12), then $\tilde{\rho}=0$ via Equations (11) and (23), and the semiaxes of the 0-ellipse cannot be defined using Equation (58). Accordingly, the 0 -ellipse coincides with the origin of the reference frame, $\left(O x_{1}\right)$, the 0 -surface of which is clearly null. For this reason, the undetermined expression, $\tilde{a}_{1}^{n-1} \rightarrow 0^{0}$ as $n=1$, appearing in Equation (93a), may safely be put equal to 0 , hence $\tilde{S}_{0}=0$, in agreement with Equation (95). On the other hand, Equations (22), (24), (25), lose their validity for $n=1$.

The $(n-1)$-surface of an infinitely thin $(n-1)$-elliptical corona can be determined by differentiating both sides of Equation (93a). The result is:

$$
\mathrm{d} \tilde{S}_{n-1}=\frac{\left[\Gamma\left(\frac{1}{2}\right)\right]^{n-1}}{\Gamma\left(1+\frac{n-1}{2}\right)} \tilde{\epsilon}_{21} \tilde{\epsilon}_{31} \cdots \tilde{\epsilon}_{n-1,1}(n-1) \tilde{a}_{1}^{n-2} \mathrm{~d} \tilde{a}_{1}
$$

which is independent of the reference frame.

In summary, the weighted mean standard deviation distribution, $f(\tilde{\chi}) \mathrm{d} \tilde{\chi}$, may be expressed as a multiple integral where the integration domain, $\tilde{\Delta}_{n}$, is an infinitely thin $n$-cylindrical corona where the axis coincides with the coordinate axis, $X_{n}$, and the semiaxes of the $(n-1)$-elliptical orthogonal section are defined by Equation (58). The result, expressed by Equation (92), after some algebra takes the form:

$f(\tilde{\chi}) \mathrm{d} \tilde{\chi}=\frac{\tilde{C}_{n}\left(n \omega_{1}^{*} \omega_{2}^{*} \cdots \omega_{n}^{*}\right)^{1 / 2}}{(2 \pi)^{(n-1) / 2} \sigma_{\tilde{m}}^{n-1}} \exp \left[-\frac{(n-1) \tilde{\chi}^{2}}{2 \sigma_{\tilde{m}}^{2}}\right] \int_{-\infty}^{+\infty} \frac{1}{\sqrt{2 \pi} \sigma_{\tilde{m}}} \frac{1}{\sqrt{n}} \exp \left(-\frac{X_{n}^{2} / n}{2 \sigma_{\tilde{m}}^{2}}\right) \mathrm{d} X_{n} \iint \cdots \int_{\tilde{\Delta}_{n-1}} \mathrm{~d} X_{1} \mathrm{~d} X_{2} \cdots \mathrm{d} X_{n-1}$

where the integration domain of the ordinary and the multiple integral are the axis and the $(n-1)$-elliptical orthogonal section, respectively, of the $n$-cylindrical corona, hence $\tilde{\Delta}_{n-1}=\mathrm{d} \tilde{S}_{n-1}$.

\section{The Solution}

The substitution of Equations (58) and (96) into (97), after long but stimulating algebra yields [6], Chapter 4:

$$
f(\tilde{\chi}) \mathrm{d} \tilde{\chi}=\frac{2}{\Gamma\left(\frac{n-1}{2}\right)} \exp \left[-\frac{(n-1) \tilde{\chi}^{2}}{2 \sigma_{\tilde{m}}^{2}}\right]\left[\left(\frac{n-1}{2}\right)^{1 / 2} \frac{\tilde{\chi}}{\sigma_{\tilde{m}}}\right]^{n-2} \mathrm{~d}\left[\left(\frac{n-1}{2}\right)^{1 / 2} \frac{\tilde{\chi}}{\sigma_{\tilde{m}}}\right]
$$

where due account has been paid to Equations (94a), (94c), together with the normalizing condition:

$$
\int_{0}^{+\infty} f(\tilde{\chi}) \mathrm{d} \tilde{\chi}=1
$$


which, after integration as outlined above via Equation (97), is equivalent to:

$$
\tilde{C}_{n}\left(n \omega_{1}^{*} \omega_{2}^{*} \cdots \omega_{n}^{*}\right)^{1 / 2} \tilde{\epsilon}_{21} \tilde{\epsilon}_{31} \cdots \tilde{\epsilon}_{n-1,1} \lambda_{1}^{-(n-1) / 2}=1
$$

according to Equation (98) that, in addition, can be related to a chi square distribution with $(n-1)$ degrees of freedom.

The distribution, $f(\tilde{\chi}) \mathrm{d} \tilde{\chi}$, expressed by Equation (98), is formally identical to its counterpart related to the arithmetic mean, $f(\bar{\chi}) \mathrm{d} \bar{\chi}$, expressed in an earlier attempt [3]. Accordingly, related results can be extended to the weighted mean provided the random variable, $\bar{\chi}$, and the rms error, $\sigma_{\bar{m}}$, are replaced by $\tilde{\chi}$ and $\sigma_{\tilde{m}}$, respectively.

The expected values, $(\tilde{\chi})^{*},\left(\tilde{\chi}^{2}\right)^{*}$, take the expression:

$$
\begin{gathered}
(\tilde{\chi})^{*}=\frac{\Gamma\left(\frac{n}{2}\right)}{\left(\frac{n-1}{2}\right)^{1 / 2} \Gamma\left(\frac{n-1}{2}\right)} \sigma_{\tilde{m}} \\
\left(\tilde{\chi}^{2}\right)^{*}=\sigma_{\tilde{m}}^{2}
\end{gathered}
$$

and the rms error of the weighted mean standard deviation distribution, $\sigma_{\tilde{\chi}}=\left[\left(\tilde{\chi}^{2}\right)^{*}-\left(\tilde{\chi}^{*}\right)^{2}\right]^{1 / 2}$, takes the expression:

$$
\sigma_{\tilde{\chi}}=\left[1-\frac{n-1}{2} \frac{\Gamma^{2}\left(\frac{n}{2}\right)}{\Gamma^{2}\left(\frac{n+1}{2}\right)}\right]^{1 / 2} \sigma_{\tilde{m}}
$$

which, for $n \gg 1$, is approximated by the asymptotic formula:

$$
\sigma_{\tilde{\chi}} \sim \frac{1}{\sqrt{2 n}} \sigma_{\tilde{m}}
$$

where the ratio, $\sigma_{\tilde{\chi}}^{2} / \sigma_{\tilde{m}}^{2}$, is understimated [3].

In summary, the weighted mean standard deviation distribution is explicitly expressed by Equation (98) and related expectation values, $(\tilde{\chi})^{*},\left(\tilde{\chi}^{2}\right)^{*}$, and rms error, $\sigma_{\tilde{\chi}}$, are expressed by Equations (101)-(103), respectively. Finally, an asymptotic formula involving $\sigma_{\tilde{\chi}}$ is shown by Equation (104), where the value is understimated. The above mentioned results, Equations (101)-(104), have the same formal expression with respect to their counterparts in the special case where the weighted mean reduces to the arithmetic mean [3].

\section{Conclusions}

The weighted mean standard deviation distribution and related parameters have been determined following a procedure where the geometrical framework is clearly shown using typical formulation generalized to $n$-spaces. After exploiting three different attempts, the integration has been performed via a change of reference frame, where the integration domain turns out to be an infinitely thin, homotetic $n$-cylindrical corona that is $(n-1)$ axially symmetric ${ }^{6}$ with respect to a coordinate axis.

In the special case where the weighted mean reduces to the arithmetic mean, the results of the current paper reduce to their counterparts (where present) in the parent paper [3]. In particular, the integration domain reduces to an infinitely thin $n$-cylindrical corona where the symmetry axis coincides with a coordinate axis and the orthogonal section is a $(n-1)$-circle. Accordingly, $(n-1)$ eigenvalues are coincident and the remaining one is null.

\footnotetext{
${ }^{6}$ In ordinary plane and space, ellipses and triaxial ellipsoids are referred to as having biaxial and triplanar symmetry, respectively. By extension to $(n-1)$-planes and $n$-spaces, $(n-1)$-ellipses and $n$-ellipsoids can be referred to as having $(n-1)$-axial and $n$-planar symmetry, respectively.
} 


\section{Acknowledgements}

A more extended version of the current attempt appears in a specific textbook [6] by the author.

\section{References}

[1] Misner, C.W., Wheeler, J.A. and Thorne, K.S. (1973) Gravitation. W.H. Freeman \& Company.

[2] Greene, B. (1999) The Elegant Universe: Superstrings, Hidden Dimensions, and the Quest for the Ultimate Theory. W.W. Norton, New York.

[3] Caimmi, R. (2013) The Arithmetic Mean Standard Deviation Distribution: A Geometrical Framework. Applied Mathematics, 4, 1-10.

[4] Malkin, Z.M. (2013) On the Calculation of Mean-Weighted Value in Astronomy. Astronomy Reports, 57, $882-887$. http://dx.doi.org/10.1134/S1063772913110048

[5] Gnedenko, B. (1978) The Theory of Probability. Mir, Moscow.

[6] Caimmi, R. (2015) Il Problema della Misura. Aracne, Roma. (In Italian, In Press)

[7] Papoulis, A. (1965) Probabilities, Random Variables, and Stochastic Processes. McGraw-Hill, New York.

[8] Smirnov, V. (1970) Cours de Mathèmatiques Supèrieures. Mir, Moscow.

[9] Gantmacher, F.R. (1966) Théorie des Matrices. Dunod, Paris.

[10] Spiegel, M.R. (1969) Mathematical Handbook of Formulas and Tables. Schaum's Outline Series, McGraw-Hill, New York. 


\section{Appendix}

\section{A. Analytic Geometry Formulation Extended to $(n+1)$-Spaces}

Analytic geometry formulation extended to $(n+1)$-spaces, used throughout the text, is outlined below. It shall be intended, but not explicitly mentioned where unnecessary, that the reference frame is $\left(O x_{1} x_{2} \cdots x_{n} \tilde{x}\right)$.

Let $r$ and $p$ be a generic straight line and $n$-plane, respectively, defined as:

$$
\begin{gathered}
r: \frac{x_{1}-x_{10}}{\ell_{1}}=\frac{x_{2}-x_{20}}{\ell_{2}}=\cdots=\frac{x_{n}-x_{n 0}}{\ell_{n}}=\frac{\tilde{x}-\tilde{x}_{0}}{\tilde{\ell}} \\
p: \sum_{i=1}^{n} a_{i} x_{i}+\tilde{a} \tilde{x}=A
\end{gathered}
$$

where $\mathbf{P}_{0} \equiv\left(x_{10}, x_{20}, \cdots, x_{n 0}, \tilde{x}_{0}\right)$ is a fixed point belonging to $r$ and $\ell_{1}, \ell_{2}, \cdots, \ell_{n}, \tilde{\ell} ; a_{1}, a_{2}, \cdots, a_{n}, \quad \tilde{a}, \mathrm{~A}$; are specified coefficients.

Let $\alpha=\widehat{r p}, 0 \leq \alpha \leq \pi / 2$, be the angle formed by the straight line and the $n$-plane. Related trigonometric functions can be inferred from the explicit expression of the sine, as:

$$
\sin \alpha=\left|\frac{\sum_{i=1}^{n} a_{i} \ell_{i}+\tilde{a} \tilde{\ell}}{\left[\sum_{i=1}^{n} a_{i}^{2}+\tilde{a}^{2}\right]^{1 / 2}\left[\sum_{i=1}^{n} \ell_{i}^{2}+\tilde{\ell}^{2}\right]^{1 / 2}}\right|
$$

which, in the case of interest $\left(\ell_{1}=\ell_{2}=\cdots=\ell_{n}=0 ; \tilde{\ell}=1 ; a_{i}=\omega_{i}^{*}, 1 \leq i \leq n ; \tilde{a}=-1 ; A=0\right)$, reduces to:

$$
\sin \alpha=\left[\frac{1}{\sum_{i=1}^{n}\left(\omega_{i}^{*}\right)^{2}+1}\right]^{1 / 2} ; \quad \cos \alpha=\left[\frac{\sum_{i=1}^{n}\left(\omega_{i}^{*}\right)^{2}}{\sum_{i=1}^{n}\left(\omega_{i}^{*}\right)^{2}+1}\right]^{1 / 2}
$$

where $r$ coincides with the coordinate axis, $\tilde{x}$, and $p$ passes through the origin.

Let $r^{\prime}$ be a generic straight line, defined as:

$$
r^{\prime}: \frac{x_{1}-x_{10}^{\prime}}{\ell_{1}^{\prime}}=\frac{x_{2}-x_{20}^{\prime}}{\ell_{2}^{\prime}}=\cdots=\frac{x_{n}-x_{n 0}^{\prime}}{\ell_{n}^{\prime}}=\frac{\tilde{x}-\tilde{x}_{0}^{\prime}}{\tilde{\ell}^{\prime}}
$$

where $\mathbf{P}_{0}^{\prime} \equiv\left(x_{10}^{\prime}, x_{20}^{\prime}, \cdots, x_{n 0}^{\prime}, \tilde{x}_{0}^{\prime}\right)$ is a fixed point belonging to $r^{\prime}$ and $\ell_{1}^{\prime}, \ell_{2}^{\prime}, \cdots, \ell_{n}^{\prime}, \tilde{\ell}^{\prime}$, are specified coefficients.

Let $\beta=\widehat{r r^{\prime}}, 0 \leq \beta \leq \pi / 2$, be the angle formed by the straight lines, $r$ and $r^{\prime}$. Related trigonometric functions can be inferred from the explicit expression of the cosine, as:

$$
\cos \beta=\left|\frac{\sum_{i=1}^{n} \ell_{i} \ell_{i}^{\prime}+\tilde{\ell}^{\prime}}{\left[\sum_{i=1}^{n} \ell_{i}^{2}+\tilde{\ell}^{2}\right]^{1 / 2}\left[\sum_{i=1}^{n}\left(\ell_{i}^{\prime}\right)^{2}+\left(\tilde{\ell}^{\prime}\right)^{2}\right]^{1 / 2}}\right|
$$

which, in the case of interest $\left(\ell_{j}=0,1 \leq j \neq i \leq n ; \quad \ell_{i}=\sqrt{n-1} \tilde{\chi} / \sqrt{\omega_{i}^{*}} ; \tilde{\ell}=\mp \sqrt{n-1} \tilde{\chi} ; \quad \ell_{i}^{\prime}=0,1 \leq i \leq n\right.$; $\tilde{\ell}^{\prime}=1$ ), reduces to:

$$
\cos \beta_{i}=\left(\frac{\omega_{i}^{*}}{1+\omega_{i}^{*}}\right)^{1 / 2} ; \quad \sin \beta_{i}=\left(\frac{1}{1+\omega_{i}^{*}}\right)^{1 / 2}
$$


where $r \equiv g_{i}$ is the generatrix, lying on the principal plane, $\left(O x_{i} \tilde{x}\right)$, of the $(n+1)$-cone, defined by Equation (24), and $r^{\prime}$ coincides with the coordinate axis, $\tilde{x}$.

The condition of parallelism between the straight line, $r$, and the $n$-plane, $p$, reads:

$$
\sum_{i=1}^{n} a_{i} \ell_{i}+\tilde{a} \tilde{\ell}=0
$$

which, in the case of interest $\left(a_{i}=\omega_{i}^{*}, 1 \leq i \leq n, \tilde{a}=-1\right)$, reduces to:

$$
\sum_{i=1}^{n} \omega_{i}^{*} \ell_{i}-\tilde{\ell}=0
$$

where $p$ is the $n$-plane, defined by Equation (21).

\section{B. Jacobi Formulae}

With regard to the quadratic form, defined by Equation (51), let the following linear forms be defined:

$$
\Xi_{i}=\sum_{k=1}^{n} a_{i k} x_{k} ; \quad 1 \leq i \leq n
$$

where $a_{i k}$ are elements of the matrix of the quadratic form:

$$
A=\left\|\begin{array}{cccc}
a_{11} & a_{12} & \cdots & a_{1 n} \\
a_{21} & a_{22} & \cdots & a_{2 n} \\
\vdots & \vdots & \ddots & \vdots \\
a_{n 1} & a_{n 2} & \cdots & a_{n n}
\end{array}\right\|
$$

and let the leading principal minors of the discriminant of the quadratic form, $\operatorname{det}(A)$, be defined as:

$$
\begin{gathered}
A_{12 \cdots k}^{(n)}= \\
=\left|\begin{array}{cccc}
a_{11} & a_{12} & \cdots & a_{1 k} \\
a_{21} & a_{22} & \cdots & a_{2 k} \\
\vdots & \vdots & \ddots & \vdots \\
a_{k 1} & a_{k 2} & \cdots & a_{k k}
\end{array}\right| ; \quad 1 \leq k \leq n \\
A^{(n)}=1 ; \quad A_{1}^{(n)}=a_{11}
\end{gathered}
$$

where $A^{(n)}$ relates to an empty (i.e. with no element) minor, whose value is conventionally assumed equal to unity. Finally, let the following linear forms be defined as:

$$
Y_{k}=\left|\begin{array}{ccccc}
a_{11} & a_{12} & \cdots & a_{1, k-1} & \Xi_{1} \\
a_{21} & a_{22} & \cdots & a_{2, k-1} & \Xi_{2} \\
\vdots & \vdots & \ddots & \vdots & \vdots \\
a_{k 1} & a_{k 2} & \cdots & a_{k, k-1} & \Xi_{k}
\end{array}\right| ; \quad 2 \leq k \leq n
$$

and let $k_{r}$ be the rank of the matrix, $A$, where $A_{1}^{(n)} \neq 0, A_{12}^{(n)} \neq 0, \cdots, A_{12 \cdots k_{r}}^{(n)} \neq 0$. Then, according to the theory of the quadratic forms, the linear forms, $Y_{k}, 1 \leq k \leq k_{r}$, are linearly independent and the Jacobi formulae hold:

$$
\begin{gathered}
c_{k}=\frac{1}{A_{12 \cdots k}^{(n)} A_{12 \cdots k-1}^{(n)}} ; \quad 1 \leq k \leq k_{r} \\
C_{k}=0 ; \quad k_{r}+1 \leq k \leq n
\end{gathered}
$$

which allow an explicit expression of Equation (60). 
For further details, an interested reader is addredded to specific textbooks (e.g., [8], Tome III, Part I, Chapter II; [9], Vol. I, Chapter 10).

\section{Reduction to Ordinary Geometry}

Aiming to get further insight on the general results related to $n$-spaces, the reduction to ordinary geometry, i.e. ordinary spaces, $n=3$, and ordinary planes, $n=2$, shall be considered in detail.

\section{C.1. Ordinary Planes}

In the special case, $n=2$, the quadratic form on the left-hand side of Equation (37) reduces to:

$$
\phi\left(x_{1}, x_{2}\right)=\omega_{1}^{*} \omega_{2}^{*}\left(x_{1}-x_{2}\right)^{2}
$$

and the related eigenvalue equation, expressed by Equation (51), reduces to:

$$
\lambda\left[\left(\frac{\lambda}{\omega_{2}^{*}}-1\right)+\left(\frac{\lambda}{\omega_{1}^{*}}-1\right)\right]=0
$$

where eigenvalues can be calculated by equating each factor to zero, using Equation (5). The result is:

$$
\lambda_{1}=2 \omega_{1}^{*} \omega_{2}^{*} ; \quad \lambda_{2}=0
$$

The canonical equation of the quadratic form, expressed by Equations (56)-(57), reduces to:

$$
\phi\left(X_{1}\right)=\lambda_{1} X_{1}^{2}
$$

and the related orthogonal transformation, defined by Equation (69), reduces to:

$$
X_{k}=b_{1 k} x_{1}+b_{2 k} X_{2} ; \quad k=1,2
$$

finally, the system of equations, expressed by Equation (77), reduces to:

$$
\left\{\begin{array}{l}
{\left[\omega_{1}^{*}\left(1-\omega_{1}^{*}\right)-\lambda_{k}\right] b_{1 k}+\omega_{1}^{*}\left(-\omega_{2}^{*}\right) b_{2 k}=0 ;} \\
\omega_{2}^{*}\left(-\omega_{1}^{*}\right) b_{1 k}+\left[\omega_{2}^{*}\left(1-\omega_{2}^{*}\right)-\lambda_{k}\right] b_{2 k}=0 .
\end{array}\right.
$$

and the substitution of Equation (121) into (124), using Equation (5), after some algebra yields:

$$
b_{11}=-b_{21} ; \quad b_{12}=b_{22}
$$

On the other hand, the condition of unit norm, expressed by Equation (78), reduces to:

$$
b_{11}^{2}+b_{21}^{2}=1 ; \quad b_{12}^{2}+b_{22}^{2}=1
$$

and the combination of Equations (125) and (126) yields:

$$
b_{11}=\frac{\mp 1}{\sqrt{2}} ; \quad b_{21}=\frac{ \pm 1}{\sqrt{2}} ; \quad b_{12}=\frac{\mp 1}{\sqrt{2}} ; \quad b_{22}=\frac{\mp 1}{\sqrt{2}}
$$

finally, the substitution of Equation (127) into (123) produces:

$$
X_{1}=\frac{\mp 1}{\sqrt{2}}\left(x_{1}-x_{2}\right) ; \quad X_{2}=\frac{\mp 1}{\sqrt{2}}\left(x_{1}+x_{2}\right)
$$

where, in addition, the substitution of Equation (128) into (122) yields Equation (120), as expected.

The coefficients and the linear forms appearing in Jacobi formulae, expressed by Equations (64) and (66), respectively, using Equation (5), reduce to:

$$
\begin{gathered}
\Omega^{(2)}=1 ; \quad \Omega_{1}^{(2)}=\omega_{1}^{*} \omega_{2}^{*} \\
Y_{1}=\omega_{1}^{*} \omega_{2}^{*}\left(x_{1}-x_{2}\right)
\end{gathered}
$$

and the canonical expression of the quadratic form, appearing on the left-hand side of Equation (67), reduces to: 


$$
\phi\left(Y_{1}\right)=\frac{Y_{1}^{2}}{\omega_{1}^{*} \omega_{2}^{*}}
$$

where, in addition, the substitution of Equation (130) into (131) yields Equation (119), as expected.

The equivalence of alternative canonical formulations, expressed by Equation (68), reduces to:

$$
\lambda_{1} X_{1}^{2}=\frac{Y_{1}^{2}}{\omega_{1}^{*} \omega_{2}^{*}}
$$

and the substitution of Equation (121) into (132) yields:

$$
Y_{1}=\sqrt{2} \omega_{1}^{*} \omega_{2}^{*} X_{1}
$$

which shows the connection between coordinates, $Y$ and $X$, with regard to the nonzero eigenvalue.

The canonical equation of the $(n-1)$-quadric, lateral $(n-1)$-surface of the $n$-cylinder, expressed by Equations (36) and (23), reduces to:

$$
\lambda_{1} X_{1}^{2}=\tilde{\rho}^{2}=\tilde{\chi}^{2}
$$

where the substitution of Equation (121) into (134) yields:

$$
X_{1}=\frac{\mp \tilde{\rho}}{\sqrt{2 \omega_{1}^{*} \omega_{2}^{*}}}=\frac{\mp \tilde{\chi}}{\sqrt{2 \omega_{1}^{*} \omega_{2}^{*}}}
$$

with regard to eigenvalue equation and, using Equation (132):

$$
\frac{Y_{1}^{2}}{\omega_{1}^{*} \omega_{2}^{*}}=\tilde{\rho}^{2}
$$

which is equivalent to:

$$
Y_{1}=\mp \sqrt{\omega_{1}^{*} \omega_{2}^{*}} \tilde{\rho}=\mp \sqrt{\omega_{1}^{*} \omega_{2}^{*}} \tilde{\chi}
$$

with regard to Jacobi formulae. In any case, the $n$-cylinder reduces to a band where the axis coincides with a coordinate axis.

\section{C.2. Ordinary Spaces}

In the special case, $n=3$, the quadratic form on the left-hand side of Equation (37) reduces to:

$$
\phi\left(x_{1}, x_{2}, x_{3}\right)=\omega_{1}^{*} \omega_{2}^{*}\left(x_{1}-x_{2}\right)^{2}+\omega_{1}^{*} \omega_{3}^{*}\left(x_{1}-x_{3}\right)^{2}+\omega_{2}^{*} \omega_{3}^{*}\left(x_{2}-x_{3}\right)^{2}
$$

and the related eigenvalue equation, expressed by Equation (51), reduces to:

$$
\lambda\left[\left(\frac{\lambda}{\omega_{2}^{*}}-1\right)\left(\frac{\lambda}{\omega_{3}^{*}}-1\right)+\left(\frac{\lambda}{\omega_{1}^{*}}-1\right)\left(\frac{\lambda}{\omega_{3}^{*}}-1\right)+\left(\frac{\lambda}{\omega_{1}^{*}}-1\right)\left(\frac{\lambda}{\omega_{2}^{*}}-1\right)\right]=0
$$

where eigenvalues can be calculated by equating each factor to zero, using Equation (5). The result is:

$$
\begin{gathered}
\lambda_{1}=\left(\omega_{2}^{*} \omega_{3}^{*}+\omega_{1}^{*} \omega_{3}^{*}+\omega_{1}^{*} \omega_{2}^{*}\right)-\sqrt{\Delta} \\
\lambda_{2}=\left(\omega_{2}^{*} \omega_{3}^{*}+\omega_{1}^{*} \omega_{3}^{*}+\omega_{1}^{*} \omega_{2}^{*}\right)+\sqrt{\Delta} \\
\lambda_{3}=0 \\
\Delta=\left(\omega_{2}^{*} \omega_{3}^{*}+\omega_{1}^{*} \omega_{3}^{*}+\omega_{1}^{*} \omega_{2}^{*}\right)-3 \omega_{1}^{*} \omega_{2}^{*} \omega_{3}^{*}
\end{gathered}
$$

where the discriminant, $\Delta$, has necessarily to be nonnegative in that eigenvalues are real in the case under discussion.

The canonical equation of the quadratic form, expressed by Equations (56) and (57), reduces to:

$$
\phi\left(X_{1}, X_{2}\right)=\lambda_{1} X_{1}^{2}+\lambda_{2} X_{2}^{2}
$$


and the related orthogonal transformation, defined by Equation (69), reduces to:

$$
X_{k}=b_{1 k} x_{1}+b_{2 k} x_{2}+b_{3 k} x_{3} ; \quad k=1,2,3
$$

finally, the system of equations, expressed by Equation (77), reduces to:

$$
\left\{\begin{array}{l}
{\left[\omega_{1}^{*}\left(1-\omega_{1}^{*}\right)-\lambda_{k}\right] b_{1 k}+\omega_{1}^{*}\left(-\omega_{2}^{*}\right) b_{2 k}+\omega_{1}^{*}\left(-\omega_{3}^{*}\right) b_{3 k}=0 ;} \\
\omega_{2}^{*}\left(-\omega_{1}^{*}\right) b_{1 k}+\left[\omega_{2}^{*}\left(1-\omega_{2}^{*}\right)-\lambda_{k}\right] b_{2 k}+\omega_{2}^{*}\left(-\omega_{3}^{*}\right) b_{3 k}=0 ; \\
\omega_{3}^{*}\left(-\omega_{1}^{*}\right) b_{1 k}+\omega_{3}^{*}\left(-\omega_{2}^{*}\right) b_{2 k}+\left[\omega_{3}^{*}\left(1-\omega_{3}^{*}\right)-\lambda_{k}\right] b_{3 k}=0 .
\end{array}\right.
$$

and the substitution of Equations (140) into (143), using Equation (5), after some algebra yields:

$$
b_{3 k}=-b_{1 k}-b_{2 k} ; \quad k=1,2
$$

On the other hand, the condition of unit norm, expressed by Equation (78), reduces to:

$$
b_{1 k}^{2}+b_{2 k}^{2}+b_{3 k}^{2}=1 ; \quad k=1,2,3
$$

and the combination of Equations (144) and (145) yields:

$$
2 b_{1 k}^{2}+2 b_{2 k}^{2}+2 b_{1 k} b_{2 k}=1 ; \quad k=1,2
$$

where $b_{2 k}$ can be expressed in terms of $b_{1 k}$ as:

$$
b_{2 k}=\frac{-b_{1 k} \mp \sqrt{2-3 b_{1 k}^{2}}}{2}
$$

and the substitution of Equation (147) into (144) after some algebra yields:

$$
b_{3 k}=\frac{-b_{1 k} \pm \sqrt{2-3 b_{1 k}^{2}}}{2}
$$

according to the condition of unit norm.

The system of equations, expressed by Equation (143), $k=1,2$, can be solved by substitution of Equations (147) and (148) into (143), but related formulation is extremely cumbersome and direct numerical computations would be preferred [6], Chapter 4.

Concerning the special case, $k=3$, the solutions of the system of equations, expressed by Equation (143), are:

$$
b_{13}=b_{23}=b_{33}
$$

where the condition of unit norm selects the solution:

$$
b_{13}=b_{23}=b_{33}=\mp \frac{1}{\sqrt{3}}
$$

which completely specifies the orthogonal transformation, defined by Equation (142).

The substitution of Equation (142) into (141), via comparison with Equation (138) term by term, after some algebra yields [6], Chapter 4:

$$
\begin{gathered}
\lambda_{1} b_{11}^{2}+\lambda_{2} b_{12}^{2}=\omega_{1}^{*} \omega_{2}^{*}+\omega_{1}^{*} \omega_{3}^{*} \\
\lambda_{1} b_{21}^{2}+\lambda_{2} b_{22}^{2}=\omega_{1}^{*} \omega_{2}^{*}+\omega_{2}^{*} \omega_{3}^{*} \\
\lambda_{1} b_{31}^{2}+\lambda_{2} b_{32}^{2}=\omega_{1}^{*} \omega_{3}^{*}+\omega_{2}^{*} \omega_{3}^{*} \\
\lambda_{1} b_{11} b_{21}+\lambda_{2} b_{12} b_{22}=-\omega_{1}^{*} \omega_{2}^{*} \\
\lambda_{1} b_{11} b_{31}+\lambda_{2} b_{12} b_{32}=-\omega_{1}^{*} \omega_{3}^{*} \\
\lambda_{1} b_{21} b_{31}+\lambda_{2} b_{22} b_{32}=-\omega_{2}^{*} \omega_{3}^{*}
\end{gathered}
$$

which have been cheched numerically for assigned choices of the input parameters, $\omega_{1}^{*}, \omega_{2}^{*}, \omega_{3}^{*}=1-\omega_{1}^{*}-\omega_{2}^{*}$. 
The coefficients and the linear forms appearing in Jacobi formulae, expressed by Equations (64) and (66), respectively, using Equation (5), reduce to:

$$
\begin{gathered}
\Omega^{(3)}=1 ; \quad \Omega_{1}^{(3)}=\omega_{1}^{*}\left(1-\omega_{1}^{*}\right) ; \quad \Omega_{2}^{(3)}=\omega_{1}^{*} \omega_{2}^{*} \omega_{3}^{*} \\
Y_{1}=\omega_{1}^{*} \omega_{2}^{*}\left(x_{1}-x_{2}\right)+\omega_{1}^{*} \omega_{3}^{*}\left(x_{1}-x_{3}\right) \\
Y_{2}=\omega_{1}^{*} \omega_{2}^{*} \omega_{3}^{*}\left(x_{2}-x_{3}\right)
\end{gathered}
$$

and the canonical expression of the quadratic form, appearing on the left-hand side of Equation (67), reduces to:

$$
\phi\left(Y_{1}, Y_{2}\right)=\frac{Y_{1}^{2}}{\omega_{1}^{*} \omega_{2}^{*}+\omega_{1}^{*} \omega_{3}^{*}}+\frac{Y_{2}^{2}}{\omega_{1}^{*} \omega_{2}^{*} \omega_{3}^{*}\left(\omega_{1}^{*} \omega_{2}^{*}+\omega_{1}^{*} \omega_{3}^{*}\right)}
$$

where, in addition, the substitution of Equations (153)-(154) into (155) yields Equation (138), as expected.

The equivalence of alternative canonical formulations, expressed by Equation (68), reduces to:

$$
\begin{gathered}
\lambda_{1} X_{1}^{2}=\frac{Y_{1}^{2}}{\omega_{1}^{*} \omega_{2}^{*}+\omega_{1}^{*} \omega_{3}^{*}} \\
\lambda_{2} X_{2}^{2}=\frac{Y_{2}^{2}}{\omega_{1}^{*} \omega_{2}^{*} \omega_{3}^{*}\left(\omega_{1}^{*} \omega_{2}^{*}+\omega_{1}^{*} \omega_{3}^{*}\right)}
\end{gathered}
$$

and the substitution of Equations (140a), (140b), into (156), (157), respectively, after some algebra yields:

$$
\begin{gathered}
Y_{1}=\left(\omega_{1}^{*} \omega_{2}^{*}+\omega_{1}^{*} \omega_{3}^{*}\right)^{1 / 2}\left(\omega_{2}^{*} \omega_{3}^{*}+\omega_{1}^{*} \omega_{3}^{*}+\omega_{1}^{*} \omega_{2}^{*}-\sqrt{\Delta}\right)^{1 / 2} X_{1} \\
Y_{2}=\left(\omega_{1}^{*} \omega_{2}^{*} \omega_{3}^{*}\right)^{1 / 2}\left(\omega_{1}^{*} \omega_{2}^{*}+\omega_{1}^{*} \omega_{3}^{*}\right)^{1 / 2}\left(\omega_{2}^{*} \omega_{3}^{*}+\omega_{1}^{*} \omega_{3}^{*}+\omega_{1}^{*} \omega_{2}^{*}+\sqrt{\Delta}\right)^{1 / 2} X_{2}
\end{gathered}
$$

which shows the connection between coordinates, $Y$ and $X$, with regard to nonzero eigenvalues.

The canonical equation of the $(n-1)$-quadric, lateral $(n-1)$-surface of a $n$-cylinder, expressed by Equations (36) and (23), reduces to:

$$
\lambda_{1} X_{1}^{2}+\lambda_{2} X_{2}^{2}=\tilde{\rho}^{2}=2 \tilde{\chi}^{2}
$$

where the substitution of Equations (140a)-(140b), into (160) yields:

$$
\left(\omega_{2}^{*} \omega_{3}^{*}+\omega_{1}^{*} \omega_{3}^{*}+\omega_{1}^{*} \omega_{2}^{*}-\sqrt{\Delta}\right) X_{1}^{2}+\left(\omega_{2}^{*} \omega_{3}^{*}+\omega_{1}^{*} \omega_{3}^{*}+\omega_{1}^{*} \omega_{2}^{*}+\sqrt{\Delta}\right) X_{2}^{2}=\tilde{\rho}^{2}=2 \tilde{\chi}^{2}
$$

with regard to eigenvalue equation and, using Equations (156)-(157):

$$
\frac{Y_{1}^{2}}{\omega_{1}^{*} \omega_{2}^{*}+\omega_{1}^{*} \omega_{3}^{*}}+\frac{Y_{2}^{2}}{\omega_{1}^{*} \omega_{2}^{*} \omega_{3}^{*}\left(\omega_{1}^{*} \omega_{2}^{*}+\omega_{1}^{*} \omega_{3}^{*}\right)}=\tilde{\rho}^{2}=2 \tilde{\chi}^{2}
$$

with regard to Jacobi formulae. In any case, the $n$-cylinder reduces to an ordinary cylinder where the axis coincides with a coordinate axis, and the orthogonal section is an ellipse whose semiaxes read:

$$
\begin{aligned}
& \tilde{a}_{1}=\frac{\sqrt{2} \tilde{\chi}}{\left(\omega_{2}^{*} \omega_{3}^{*}+\omega_{1}^{*} \omega_{3}^{*}+\omega_{1}^{*} \omega_{2}^{*}-\sqrt{\Delta}\right)^{1 / 2}} \\
& \tilde{a}_{2}=\frac{\sqrt{2} \tilde{\chi}}{\left(\omega_{2}^{*} \omega_{3}^{*}+\omega_{1}^{*} \omega_{3}^{*}+\omega_{1}^{*} \omega_{2}^{*}+\sqrt{\Delta}\right)^{1 / 2}}
\end{aligned}
$$

with regard to eigenvalue equation, and: 


$$
\begin{gathered}
\tilde{a}_{2}=\sqrt{2}\left(\omega_{1}^{*} \omega_{2}^{*}+\omega_{1}^{*} \omega_{3}^{*}\right)^{1 / 2} \tilde{\chi} \\
\tilde{a}_{2}=\sqrt{2}\left(\omega_{1}^{*} \omega_{2}^{*} \omega_{3}^{*}\right)^{1 / 2}\left(\omega_{1}^{*} \omega_{2}^{*}+\omega_{1}^{*} \omega_{3}^{*}\right)^{1 / 2} \tilde{\chi}
\end{gathered}
$$

with regard to Jacobi formulae.

\section{Corrigendum [3]}

Additional help to an interested reader could arise from the following corrigendum to a quoted earlier attempt devoted to the arithmetic mean [3].

- p. 2, right column: Equations (9) and (10) are inferred via Equation (8) instead of Equation (6).

- p. 3, left column: the extension of usual formulation of analytic geometry has to be intended to $(n+1)$ spaces instead of $n$-spaces.

- p. 3, right column: Equation (18) relates to the polar intead of equatorial semiaxis.

- p. 4, right column: Equation (30) relates to a $n$-hyperboloid intead of a $(n+1)$-hyperboloid, where $n$ hyperboloids are to be intended as $n$-surfaces instead of $n$-volumes.

- p. 4, right column: Equation (30) relates to the polar intead of equatorial semiaxis.

- p. 4, right column: the intersection, $i_{p}$, expressed by Equation (31), is to be conceived as a $(n-1)$-line intead of a $(n-1)$-surface.

- p. 5, left column: $X_{1}, X_{2}, \cdots, X_{n}$, are to be conceived as principal axes of a $n$-surface instead of a $n$ volume.

- p. 5, left column: to avoid ambiguity, the direction cosines should be defined as $\gamma_{\imath \mathrm{k}}=\widehat{X_{\ell} X_{k}}$ with regard to the starting reference frame, $\left(O x_{1} x_{2} \cdots x_{n}\right) ; \Gamma_{k \ell}=\widehat{x_{k} X_{\ell}}$ with regard to the resulting reference frame, $\left(O X_{1} X_{2} \cdots X_{n}\right)$. Accordingly, $\gamma_{k \ell}$ has to be replaced by $\Gamma_{k \ell}$ in Equations (32), (33), (36), (37), and $\gamma_{j \ell}$ has to be replaced by $\Gamma_{j \ell}$ in Equation (37). Finally, $\gamma$ has to be replaced by $\Gamma$ everywhere in Equation (38).

- p. 6, left column: the integration domain, $\tilde{D}_{n}$, represents an infinitely thin $n$-cylindrical corona instead of a $n$-cylinder.

- p. 8, left column: $\tilde{\Delta}_{n-1}=d \tilde{S}_{n-1}$ has to be considered instead of $\tilde{\Delta}_{n-1}=\tilde{S}_{n-1}$.

- p. 8, left column: Equation (49) has to be considered instead of (18) for determining Equation (60). 